\title{
A Multiperiod Equilibrium Pricing Model
}

\author{
Minsuk Kwak, ${ }^{1}$ Traian A. Pirvu, ${ }^{1}$ and Huayue Zhang $^{2}$ \\ ${ }^{1}$ Department of Mathematics and Statistics, McMaster University, 1280 Main Street West, Hamilton, ON, Canada L8S 4 K1 \\ ${ }^{2}$ Department of Finance, Nankai University, 94 Weijin Road, Tianjin 300071, China
}

Correspondence should be addressed to Minsuk Kwak; minsuk.kwak@gmail.com

Received 7 November 2013; Revised 15 January 2014; Accepted 16 January 2014; Published 5 March 2014

Academic Editor: Pu-yan Nie

Copyright (C) 2014 Minsuk Kwak et al. This is an open access article distributed under the Creative Commons Attribution License, which permits unrestricted use, distribution, and reproduction in any medium, provided the original work is properly cited.

\begin{abstract}
We propose an equilibrium pricing model in a dynamic multiperiod stochastic framework with uncertain income. There are one tradable risky asset (stock/commodity), one nontradable underlying (temperature), and also a contingent claim (weather derivative) written on the tradable risky asset and the nontradable underlying in the market. The price of the contingent claim is priced in equilibrium by optimal strategies of representative agent and market clearing condition. The risk preferences are of exponential type with a stochastic coefficient of risk aversion. Both subgame perfect strategy and naive strategy are considered and the corresponding equilibrium prices are derived. From the numerical result we examine how the equilibrium prices vary in response to changes in model parameters and highlight the importance of our equilibrium pricing principle.
\end{abstract}

\section{Introduction}

Hitherto, there has been an increasing literature on pricing contingent claims written on nontradable underlyings in a dynamic multiperiod equilibrium framework. One example of such contingent claim is a weather derivative, in which case the underlying is the temperature process. One approach in pricing this financial instrument is to use a multiperiod stochastic equilibrium model. In financial economics there is a huge amount of literature on this issue. Rubinstein [1] considers a multiperiod state-preference equilibrium model without explicit modeling of production/investment. Brennan [2] looks at a multiperiod equilibrium problem in which the representative agent exhibits constant risk aversion. Bhattacharya [3] extends the model of Rubinstein [1] to show that risk/return tradeoffs are linear relations linking instantaneous expected returns of assets to the instantaneous covariance of returns with aggregate consumption. Bizid and Jouini [4] derive restrictions on the equilibrium state-price deflator independent of the choices of utility function in an incomplete market. Câmara [5] obtains preference-free option prices in a discrete equilibrium model where representative agent has exponential utility and aggregate wealth together with the underlying asset price has transformed normal distributions.
Our paper presents a partial equilibrium model with two exogenous assets, one tradable and one nontradable. The goal is to find the price of a derivative security written on tradable and nontradable underlyings in equilibrium. We assume that the tradable asset is priced in a different market so its price is exogenously given. Moreover the interest rate risk is not considered because we take the money market account as numeraire. The derivative security, written on the tradable asset and nontradable underlying, is priced in equilibrium by a representative agent who receives (unspanned) random income within an incomplete multiperiod market. The optimal strategies are obtained by backward induction. First order conditions together with the market clearing give the equilibrium prices.

Cao and Wei [6], Lee and Oren [7, 8], and Cheridito et al. [9] are related to our work. Cao and Wei [6] generalize the model of Lucas [10] to provide an equilibrium framework for valuing weather derivatives in a multiperiod setting. Lee and Oren [7] explore a single-period equilibrium pricing model in a multicommodity setting and mean-variance preferences. Lee and Oren [8] made a followup in a multiperiod framework. Cheridito et al. [9] establish results on the existence and uniqueness of equilibrium in dynamically incomplete financial markets with preferences of monetary 
type and heterogeneous agents. Utility indifference pricing is also a way for pricing derivatives written on nontradable underlying. The utility indifference price was introduced by Hodges and Neuberger [11]. By now, there are several papers on this topic; we recall only a few (on discrete time Musiela et al. [12]; on continuous time Henderson [13]; Musiela and Zariphopoulou [14]; for an overview see Henderson and Hobson [15]). Pirvu and Zhang [16] derive utility indifference prices in a model with time changing risk aversion.

In our model the representative agent has risk preferences of exponential type with time and state-dependent coefficient of risk aversion. Inspired by Gordon and St-Amour [17], we assume that the representative agent's risk aversion coefficient is a stochastic process. Gordon and St-Amour [17] motivate this change by the fact that stochastic risk aversion can explain asset-price movements which cannot be explained by constant risk aversion. Lately, the issue of time changing risk aversion received some attention in the financial literature. For instance, Barberis and Huang [18] consider a model in which the loss aversion depends on prior gains and losses, so it may change through time. Danthine et al. [19] allow the representative agent's coefficient of relative risk aversion to vary with the underlying economy's growth rate. Gordon and St-Amour [20] explain equity premium puzzle by statedependent risk preferences. Yuan and Chen [21] show that dynamic risk aversion plays a critical role in the dynamics of asset price fluctuations. Recently, Berrada et al. [22] consider growth regime-dependent risk aversion of representative agent and examine the effect of regime-dependent preference on the structure of equilibrium.

A time changing risk preference leads to time inconsistent investment strategies. It means that an investor may have an incentive to deviate from the classical optimal strategy which he/she computed at some past time. Let us define the naive strategies as the classical optimal strategies given that the agent does not update his/her risk preferences. The naive strategies cannot be implemented in the absence of a precommitment. In order to overcome the time inconsistency issue, Pirvu and Zhang [16] and Björk and Murgoci [23] develop a theory for stochastic control problems which are time inconsistent in the sense that they do not admit the Bellman optimality principle; they consider the subgame perfect equilibrium as a substitute for the Bellman optimality principle. In $\mathrm{Wu}$ [24], subgame perfect equilibrium strategies are studied for a multiperiod mean-variance portfolio selection problem with wealth-dependent risk aversion. In our paper, we follow Pirvu and Zhang [16] in introducing subgame perfect strategies. Subgame perfect strategies denote the strategies obtained from the subgame perfect equilibrium. The corresponding equilibrium price obtained by imposing the market clearing condition with subgame perfect strategies of representative agent is called as subgame perfect equilibrium price. On the other hand, market clearing condition with naive strategies gives naive equilibrium price. The naive equilibrium price is considered as a benchmark price. By comparing the subgame perfect equilibrium price and the naive equilibrium price, the difference and the importance of subgame perfect equilibrium price are highlighted.
The exogenous assets in our model may have stochastic drifts and volatilities and the assets may be correlated. A derivative security is priced in equilibrium within this model. Our main result is an iterative algorithm which yields the subgame perfect equilibrium prices. At each stage the equilibrium prices depend on the current risk aversion level and all previous wealth and risk aversion levels. The algorithm constructs recursively one period pricing kernels. Moreover, the naive equilibrium pricing kernel equals the marginal utility. We prove that the equilibrium pricing measures are martingale measures so the equilibrium prices are arbitrage free.

Numerical experiments shed light into the importance of the pricing principle to find subgame perfect equilibriums price. For the numerical result, we consider a two-state regime switching model (bull and bear markets) and assume that the representative agent becomes more risk averse in the bear market. The result shows that the equilibrium prices (both subgame perfect equilibrium price and naive equilibrium price) are decreasing in risk aversion. It's because the derivative can be regarded as a risky asset so agent's demand for the derivative is reduced if he/she is more risk averse. Since the risk aversions of the agent in two states are different, we may try to approximate the subgame perfect equilibrium price with a new risk aversion, say average risk aversion which is a weighted average of two risk aversion (risk aversions in bull market and bear market) with the steady state probabilities of two states as the weights. However, we find that there is significant gap between the exact subgame perfect equilibrium price and the naive equilibrium price with average risk aversion if the risk aversion in the bear market is much higher than the risk aversion in the bull market. This means that the naive equilibrium price with average risk aversion cannot be a useful estimate of subgame perfect equilibrium price especially when the agent's preference changes a lot depending on the market environment.

We also examine the effect of the number of units of derivatives in the market on the equilibrium prices and we find out that the equilibrium prices are decreasing in number of units of derivatives. This result is consistent with Gârleanu et al. [25] which examine the effects of demand pressure on option prices. Moreover, it is observed that the gap between the naive equilibrium price with average risk aversion and the subgame perfect equilibrium price gets larger if the number of units of derivatives is large enough. Hence the naive equilibrium price is not a good estimate of the subgame perfect equilibrium price in this case.

The remainder of this paper is organized as follows. Section 2 presents the model. Section 3 provides the equilibrium pricing valuation. Numerical results are presented in the Section 4. Proofs of the results are delegated to an Appendices $\mathrm{A}, \mathrm{B}$, and $\mathrm{C}$.

\section{The Model}

We consider a multi-period stochastic model of investment. The trading horizon is $[0, T]$, with $T$ an exogenous finite horizon. There are $N+1$ trading dates: $t_{n}=n h$, for $n=0,1, \ldots, N$, 
and $h=T / N$. Let $\left(b^{1}, b^{2}, \ldots, b^{d}\right):=\left(b_{t_{n}}^{1}, b_{t_{n}}^{2}, \ldots, b_{t_{n}}^{d}\right)_{n=0,1, \ldots, \infty}$, be a $d$-dimensional binomial random walk on a complete probability space $\left(\Omega, \mathscr{F},\left\{\mathscr{F}_{t_{n}}\right\}, \mathbb{P}\right)$. The random walk is assumed to be symmetric under $\mathbb{P}$ in the sense that

$$
\mathbb{P}\left(\Delta b_{t_{n}}^{i}= \pm 1\right)=\frac{1}{2}, \quad i=1,2, \ldots, d .
$$

There are three securities available for trading in our model; a money market account, a primary asset (e.g., stock or commodity) and a derivative security. We take the money market account as numeraire, thus it can be assumed to offer zero interest rate. The primary asset price process $C:=$ $\left\{C_{t_{n}} ; n=0,1, \ldots, N\right\}$, follows the difference equation:

$$
\begin{gathered}
\Delta C_{t_{n}}=C_{t_{n}}\left(\mu_{t_{n}}^{c} h+\sigma_{t_{n}}^{c} \sqrt{h} \Delta b_{t_{n}}^{1}\right), \quad n=0,1, \ldots, N-1, \\
C_{0}=c>0,
\end{gathered}
$$

for some adapted drift process $\mu^{c}:=\left\{\mu_{t}^{c} ; t=0, h, \ldots,(N-\right.$ 1) $h, N h\}$ and volatility process $\sigma^{c}:=\left\{\sigma_{t}^{c} ; t=0, h, \ldots,(N-\right.$ 1) $h, N h\}$ which are chosen so that the commodity price remains positive. The derivative security $D:=\left\{D_{t_{n}} ; n=\right.$ $0,1, \ldots, N\}$ is written on the primary asset $C$ and a nontradable underlying $S:=\left\{S_{t_{n}} ; n=0,1, \ldots, N\right\}$ which follows the difference equation:

$$
\begin{gathered}
\Delta S_{t_{n}}=S_{t_{n}}\left(\mu_{t_{n}}^{s} h+\sigma_{t_{n}}^{s} \sqrt{h}\left(\rho \Delta b_{t_{n}}^{1}+\sqrt{1-\rho^{2}} \Delta b_{t_{n}}^{2}\right)\right), \\
n=0,1, \ldots, N-1, \\
S_{0}=s>0,
\end{gathered}
$$

for some adapted drift process $\mu^{s}:=\left\{\mu_{t}^{s} ; t=0, h, \ldots,(N-\right.$ 1) $h, N h\}$, volatility process $\sigma^{s}:=\left\{\sigma_{t}^{s} ; t=0, h, \ldots,(N-\right.$ 1) $h, N h\}$, and a correlation coefficient $\rho$, with $|\rho|<1$. The two-dimensional process $P=(C, S)$, exogenously given, is referred to as the forward process.

Since the derivative security $D$ is to be priced in equilibrium, we have a partial equilibrium model. It is motivated by a situation in which the primary asset and derivative are priced in different markets. As an example, consider energy market and weather derivatives. Although the energy price and weather derivatives are correlated (in California, a high correlation between energy prices and temperature process was observed), energy prices and weather derivative prices are determined in different markets.

2.1. Trading Strategies. Let $\alpha_{t_{n}}$ be the amount invested in the primary asset at time $t_{n}$, and let $\beta_{t_{n}}$ be the number of shares of derivative held at time $t_{n}$; denote $\pi_{t_{n}}:=\left\{\alpha_{t_{n}}, \beta_{t_{n}}\right\} \in \mathscr{F}_{t_{n}}, n=$ $0,1, \ldots, N-1$. The value of a self-financed portfolio satisfies the following stochastic difference equation:

$$
\Delta X_{t_{n}}^{\pi}:=\alpha_{t_{n}}\left(\mu_{t_{n}}^{c} h+\sigma_{t_{n}}^{c} \sqrt{h} \Delta b_{t_{n}}^{1}\right)+\beta_{t_{n}} \Delta D_{t_{n}} .
$$

At maturity, $t_{N}:=T=N h$, the representative agent in this economy receives random income $I_{t_{N}}$, which is $\mathscr{F}_{T}$ adapted. Thus, his/her final wealth is

$$
W_{t_{N}}^{\pi}=X_{t_{N}}^{\pi}+I_{t_{N}}
$$

The random income may depend on all the random walks $\left\{b^{1}, b^{2}, \ldots, b^{d}\right\}$, so it may not be spanned by the existing assets.

2.2. Risk Preferences. The representative agent utility is assumed to be of exponential type, time, and state dependent. The coefficient of absolute risk aversion is a stochastic process $\gamma_{t_{n}}, \mathscr{F}_{t_{n}}$ adapted, $n=0,1, \ldots, N-1$. More precisely,

$$
U\left(x, t_{n}, \omega\right)=-\exp \left(-\gamma_{t_{n}}(\omega) x\right), \quad \omega \in \Omega .
$$

As we pointed out in Section 1, this modeling approach is not new and consistent with a number of papers that consider time changing, state-dependent risk aversion. The performance of an investment strategy $\pi$ is measured by the expected utility criterion applied to the final wealth; that is, the optimization criterion at time $t_{n}$ is given by

$$
\sup _{\pi \in \Pi_{t_{n}}} \mathbb{E}\left[-e^{-\gamma_{t_{n}} W_{t_{N}}^{\pi}} \mid \mathscr{F}_{t_{n}}\right]
$$

Here $n=0,1, \ldots, N-1, W_{t_{N}}^{\pi}$ is given by (5), and $\Pi_{t_{n}}$ denotes the set of admissible trading strategies:

$$
\begin{aligned}
\Pi_{t_{n}}:=\left\{\pi_{t_{n}}, \pi_{t_{n+1}}, \ldots, \pi_{t_{N-1}}: \pi_{t_{k}} \in \mathscr{F}_{t_{k}},\right. \\
\left.\quad \text { such that } \mathbb{E}\left|X_{t_{k}}^{\pi}\right|<\infty, k=n, n+1, \ldots, N-1\right\} .
\end{aligned}
$$

2.3. Naive Strategies. The naive strategies are the classical optimal strategies given that the risk preferences are not updated. More precisely $\hat{\pi} \in \Pi_{t_{0}}$ is a naive strategy if it satisfies

$$
\widehat{\pi}=\arg \sup _{\pi \in \Pi_{t_{0}}} \mathbb{E}\left[-\exp \left(-\gamma_{t_{0}} W_{t_{N}}^{\pi}\right) \mid \mathscr{F}_{t_{0}}\right] .
$$

They fail to remain optimal at later times $t_{n}$, in the sense that

$$
\widehat{\pi} \neq \arg \sup _{\pi \in \Pi_{t_{n}}} \mathbb{E}\left[-\exp \left(-\gamma_{t_{n}} W_{t_{N}}^{\pi}\right) \mid \mathscr{F}_{t_{n}}\right] .
$$

2.4. Subgame Perfect Strategies. In this section, we introduce the subgame perfect strategies by following Pirvu and Zhang [16]. First, let us consider the time period $[(N-1) h, N h]$ (recall that $\left.t_{N}=T=N h\right)$. At time $(N-1) h$ consider the optimization problem:

$$
\sup _{\pi \in \Pi_{t_{N-1}}} \mathbb{E}\left[-\exp \left(-\gamma_{t_{N-1}} W_{t_{N}}^{\pi}\right) \mid \mathscr{F}_{t_{N-1}}\right] .
$$

In our model sup in $\left(\mathrm{P}_{1}\right)$ is attained and we denote

$$
\pi_{t_{N-1}}^{*}=\arg \max _{\pi \in \Pi_{t_{N-1}}} \mathbb{E}\left[-\exp \left(-\gamma_{t_{N-1}} W_{t_{N}}^{\pi}\right) \mid \mathscr{F}_{t_{N-1}}\right] .
$$

On the time period $[(N-2) h, N h]$, consider trading strategies $\pi$ be of the form:

$$
\pi= \begin{cases}\pi_{t_{N-1}}^{*}, & \text { on }[(N-1) h, N h), \\ \pi_{t_{N-2}}, & \text { on }[(N-2) h,(N-1) h),\end{cases}
$$


for an arbitrary $\mathscr{F}_{t_{N-2}}$ adapted control $\pi_{t_{N-2}}$ such that $\left(\pi_{t_{N-2}}, \pi_{t_{N-1}}^{*}\right) \in \Pi_{t_{N-2}}$; consider the optimization problem

$$
\sup _{\pi \in \Pi_{t_{N-2}}} \mathbb{E}\left[-\exp \left(-\gamma_{t_{N-2}} W_{t_{N}}^{\pi}\right) \mid \mathscr{F}_{t_{N-2}}\right] .
$$

In our model sup in $\left(\mathrm{P}_{2}\right)$ is attained and we denote

$$
\left(\pi_{t_{N-2}}^{*}, \pi_{t_{N-1}}^{*}\right)=\arg \max _{\pi \in \Pi_{t_{N-2}}} \mathbb{E}\left[-\exp \left(-\gamma_{t_{N-2}} W_{t_{N}}^{\pi}\right) \mid \mathscr{F}_{t_{N-2}}\right] .
$$

Further we proceed iteratively. On the time period [ $(N-$ $n) h, N h$ ) one restricts to trading strategies $\pi$ of the form:

$$
\pi= \begin{cases}\pi_{t_{k}}^{*}, & \text { for } k=N-(n-1), N-(n-2), \ldots, N-1, \\ \pi_{t_{k}}, & \text { for } k=N-n,\end{cases}
$$

for an arbitrary $\mathscr{F}_{t_{N-n}}$ adapted control $\pi_{t_{N-n}}$ such that $\left(\pi_{t_{N-n}}\right.$, $\left.\pi_{t_{k}}^{*}\right)_{\{k=N-n+1, \ldots, N-1\}} \in \Pi_{t_{N-n}}$. Consider the optimization problem

$$
\max _{\pi \in \Pi_{t_{N-n}}} \mathbb{E}\left[-\exp \left(-\gamma_{t_{N-n}} W_{t_{N}}^{\pi}\right) \mid \mathscr{F}_{t_{N-n}}\right]
$$

The sup in $\left(\mathrm{P}_{n}\right)$ is attained and we denote

$$
\begin{aligned}
& \left(\pi_{t_{N-n}}^{*}, \pi_{t_{N-n+1}}^{*}, \ldots, \pi_{t_{N-1}}^{*}\right) \\
& \quad=\arg \max _{\pi \in \Pi_{t_{N-n}}} \mathbb{E}\left[-\exp \left(-\gamma_{t_{N-n}} W_{t_{N}}^{\pi}\right) \mid \mathscr{F}_{t_{N-n}}\right] .
\end{aligned}
$$

The subgame perfect strategy is $\pi^{*}=\left(\pi_{t_{0}}^{*}, \pi_{t_{1}}^{*}, \ldots, \pi_{t_{N}}^{*}\right)$.

2.5. Subgame Perfect Strategies versus Naive Strategies. Let us recall that time inconsistency in this model is due to time changing, state-dependent risk aversion. Indeed in the case of constant risk aversion, the subgame perfect strategies and the naive strategies coincide; that is, $\pi^{*}=\widehat{\pi}$. In general, it is hard to show that the subgame perfect strategy outperforms the naive strategy. We show that this is the case if the risk preferences are updated in the following two period models. Let us assume that $I_{t_{2}}=0$, and only one asset is available for trading (the primary asset with constant drift and volatility). It is claimed that

$$
\mathbb{E}\left[-\exp \left(-\gamma_{t_{1}} W_{t_{2}}^{\pi^{*}}\right) \mid \mathscr{F}_{t_{1}}\right] \geq \mathbb{E}\left[-\exp \left(-\gamma_{t_{1}} W_{t_{2}}^{\widehat{\pi}}\right) \mid \mathscr{F}_{t_{1}}\right] .
$$

Indeed in this model it can be shown that $\pi_{t_{1}}^{*}=\widehat{\pi}_{t_{1}}$, and hence $W_{t_{1}}^{\pi^{*}}=W_{t_{1}}^{\hat{\pi}}$. By the definition of the subgame perfect strategies, we have (16).

\section{Equilibrium Valuation}

We assume that there exists a representative agent with risk preferences given by (6). The representative agent trades $C$ and $D$ in order to maximize the expected utility of his/her final wealth. This can be achieved by the naive strategy $\widehat{\pi}=$ $(\widehat{\alpha}, \widehat{\beta})$ if the representative agent does not update his/her risk preferences. Otherwise, the subgame perfect strategy $\pi^{*}=$ $\left(\alpha^{*}, \beta^{*}\right)$ will be used. Thus, depending on whether or not the representative agent updates his/her risk preferences, we introduce two notions of equilibrium prices: subgame perfect equilibrium price and naive equilibrium price. They are given by the market clearing condition in the formal definition below.

Definition 1. Given the terminal payoff $D_{t_{N}}$ and $m_{D}>0$ units of derivative, $D_{t_{n}}$ is the subgame perfect equilibrium price if and only if

$$
\beta_{t_{n}}^{*}=m_{D}
$$

for every $n=0,1, \ldots, N-1$. Likewise, $D_{t_{n}}$ is the naive equilibrium price if and only if

$$
\widehat{\beta}_{t_{n}}=m_{D}
$$

for every $n=0,1, \ldots, N-1$.

This simply says that there is $m_{D}$ units of derivative in the market and it is priced such that "it is optimal" for the representative agent to acquire it. The interest of the representative agent in holding the derivative comes from the fact that the primary asset is correlated with the nontradable asset which is an underlying of the derivative. Moreover, agent's income is assumed to be related to the nontradable asset $S$; thus, the risk of income fluctuations due to $S$ can be hedged by trading $D$. Let $r_{t_{n}}^{c}$, given by

$$
r_{t_{n}}^{c}:=\frac{\mu_{t_{n}}^{c}}{\sigma_{t_{n}}^{c}}
$$

be the market price of risk (MPR) for the primary asset which is assumed positive. We choose time length $h$ small enough such that $1 \geq r_{t_{n}}^{c} \sqrt{h}$.

Remark 2. For the naive equilibrium price to be implemented the representative agent has to exhibit the same risk aversion throughout time. This may not be realistic since different factors (e.g., economic and financial) may change the risk preference of the representative agent. In the light of this, the naive equilibrium price can be seen as a benchmark price. A more realistic price is the subgame perfect equilibrium price; this, unlike the naive equilibrium price, takes into account the changes of the representative agent's risk aversion.

3.1. Single Period. Define $A_{t_{N-1}}:=\left\{\omega \in \Omega: \Delta b_{t_{N-1}}^{1}=1\right\}$ and $A_{t_{N-1}}^{c}:=\left\{\omega \in \Omega: \Delta b_{t_{N-1}}^{1}=-1\right\}$.

Theorem 3. The subgame perfect equilibrium price at time $t_{N-1}$ is given by

$$
D_{t_{N-1}}=\mathbb{E}^{\mathbb{Q}^{*}}\left[D_{t_{N}} \mid \mathscr{F}_{t_{N-1}}\right]
$$

where the probability measure $\mathbb{Q}^{*}$ is defined by

$$
\frac{d \mathbb{Q}^{*}}{d \mathbb{P}}=\Lambda_{t_{N}}^{*} \mathbb{E}\left[\frac{d \mathbb{Q}^{*}}{d \mathbb{P}} \mid \mathscr{F}_{t_{N-1}}\right] \text {. }
$$


The pricing kernel $\Lambda_{t_{N}}^{*}$ is

$$
\Lambda_{t_{N}}^{*}:=\left\{\begin{array}{c}
\lambda_{t_{N-1}} \frac{e^{-\gamma_{t_{N-1}}\left(m_{D} D_{t_{N}}+I_{t_{N}}\right)}}{\mathbb{E}\left[e^{-\gamma_{t_{N-1}}\left(m_{D} D_{t_{N}}+I_{t_{N}}\right)} \mid A_{t_{N-1}} \vee \mathscr{F}_{t_{N-1}}\right]}, \\
\text { if } \omega \in A_{t_{N-1}} \\
\lambda_{t_{N-1}} \frac{e^{-\gamma_{t_{N-1}}\left(m_{D} D_{t_{N}}+I_{t_{N}}\right)}}{\mathbb{E}\left[e^{-\gamma_{t_{N-1}}\left(m_{D} D_{t_{N}}+I_{t_{N}}\right)} \mid A_{t_{N-1}}^{c} \vee \mathscr{F}_{t_{N-1}}\right]}, \\
\text { if } \omega \in A_{t_{N-1}}^{c},
\end{array}\right.
$$

with

$$
\lambda_{t_{N-1}}= \begin{cases}1-r_{t_{N-1}}^{c} \sqrt{h}, & \text { if } \omega \in A_{t_{N-1}} \\ 1+r_{t_{N-1}}^{c} \sqrt{h}, & \text { if } \omega \in A_{t_{N-1}}^{c}\end{cases}
$$

The optimal trading strategy is given by

$$
\begin{aligned}
\alpha_{t_{N-1}}^{*}= & \frac{1}{2 \gamma_{t_{N-1}} \sigma_{t_{N-1}}^{c} \sqrt{h}} \log \left(\frac{1+r_{t_{N-1}}^{c} \sqrt{h}}{1-r_{t_{N-1}}^{c} \sqrt{h}}\right) \\
& +\frac{1}{2 \gamma_{t_{N-1}} \sigma_{t_{N-1}}^{c} \sqrt{h}} \\
& \times \log \left(\frac{\mathbb{E}\left[e^{-\gamma_{t_{N-1}}\left(m_{D} D_{t_{N}}+I_{t_{N}}\right)} \mid A_{t_{N-1}} \vee \mathscr{F}_{t_{N-1}}\right]}{\mathbb{E}\left[e^{-\gamma_{t_{N-1}}\left(m_{D} D_{t_{N}}+I_{t_{N}}\right)} \mid A_{t_{N-1}}^{c} \vee \mathscr{F}_{t_{N-1}}\right]}\right) .
\end{aligned}
$$

Proof. Proof of this theorem is done in Appendix A.

Remark 4. For the naive equilibrium price just replace $\gamma_{t_{N-1}}$ with $\gamma_{t_{0}}$ in the above formulas.

Next we prove that the probability measure $\mathbb{Q}^{*}$ is a martingale measure so the subgame perfect equilibrium price is arbitrage-free (similarly it can be shown that the naive equilibrium price is arbitrage free).

Lemma 5. The tradable assets $\left\{C_{t_{n}}\right\}_{n=N-1, N}$ and $\left\{D_{t_{n}}\right\}_{n=N-1, N}$ are martingales under $\mathbb{Q}^{*}$.

Proof. $\left\{D_{t_{n}}\right\}_{n=N-1, N}$ is martingale under $\mathbb{Q}^{*}$ by definition. Next we show that $\left\{C_{t_{n}}\right\}_{n=N-1, N}$ is martingale under $\mathbb{Q}^{*}$. It suffices to prove that with $n=N-1$

$$
\begin{aligned}
\mathbb{E}^{\mathbb{Q}^{*}} & {\left[\frac{C_{t_{n+1}}}{C_{t_{n}}} \mid \mathscr{F}_{t_{n}}\right] } \\
& =\mathbb{E}^{\mathbb{Q}^{*}}\left[1+\sigma_{t_{n}}^{c} \sqrt{h}\left(r_{t_{n}}^{c} \sqrt{h}+\Delta b_{t_{n}}^{1}\right) \mid \mathscr{F}_{t_{n}}\right] \\
& =1+\sigma_{t_{n}}^{c} \sqrt{h} \mathbb{E}^{\mathbb{Q}^{*}}\left[\left(r_{t_{n}}^{c} \sqrt{h}+\Delta b_{t_{n}}^{1}\right) \mid \mathscr{F}_{t_{n}}\right] \\
& =1 .
\end{aligned}
$$

This is the case if

$$
\mathbb{E}^{\mathbb{Q}^{*}}\left[\left(r_{t_{n}}^{c} \sqrt{h}+\Delta b_{t_{n}}^{1}\right) \mid \mathscr{F}_{t_{n}}\right]=0 .
$$

When $n=N-1,(26)$ is equivalent to

$$
\begin{aligned}
& \frac{r_{t_{N-1}}^{c} \sqrt{h}+1}{2} \mathbb{E}\left[\Lambda_{t_{N}}^{*} \mathbb{E}\left[\frac{d \mathbb{Q}^{*}}{d \mathbb{P}} \mid \mathscr{F}_{t_{N-1}}\right] \mid A_{t_{N-1}} \vee \mathscr{F}_{t_{N-1}}\right] \\
& +\frac{r_{t_{N-1}}^{c} \sqrt{h}-1}{2} \mathbb{E}\left[\Lambda_{t_{N}}^{*} \mathbb{E}\left[\frac{d \mathbb{Q}^{*}}{d \mathbb{P}} \mid \mathscr{F}_{t_{N-1}}\right] \mid A_{t_{N-1}}^{c} \vee \mathscr{F}_{t_{N-1}}\right] \\
& =\frac{1-\left(r_{t_{N-1}}^{c}\right)^{2} h}{2} \mathbb{E}\left[\frac{d \mathbb{Q}^{*}}{d \mathbb{P}} \mid \mathscr{F}_{t_{N-1}}\right] \\
& +\frac{\left(r_{t_{N-1}}^{c}\right)^{2} h-1}{2} \mathbb{E}\left[\frac{d \mathbb{Q}^{*}}{d \mathbb{P}} \mid \mathscr{F}_{t_{N-1}}\right]=0,
\end{aligned}
$$

and this completes the proof.

3.2. Multiple Periods. Let us define the sets $A_{t_{\mathrm{N}-n}}:=\{\omega \in$ $\left.\Omega: \Delta b_{t_{N-n}}^{1}=1\right\}$ and $A_{t_{N-n}}^{c}:=\left\{\omega \in \Omega: \Delta b_{t_{N-n}}^{1}=-1\right\}$; to ease notations, let us denote

$$
\mathbb{E}_{t_{n}}[\cdot]:=\mathbb{E}\left[\cdot \mid \mathscr{F}_{t_{n}}\right], \quad \mathbb{E}_{t_{n}}[\cdot \mid \mathscr{G}]:=\mathbb{E}\left[\cdot \mid \mathscr{G} \vee \mathscr{F}_{t_{n}}\right],
$$

for every $\mathscr{G} \subset \mathscr{F}$. The equilibrium prices are computed by a recursive algorithm. Imagine that the equilibrium prices after $t_{N-n}$ were found and now we want to find the equilibrium price at $t_{N-n}$. At time $t_{N-n}$, define the random variables $Y_{t_{N-n+1}}^{*}$ and $\widehat{Y}_{t_{N-n+1}}$ by

$$
\begin{aligned}
e^{-\gamma_{t_{N-n}} Y_{t_{N-n+1}}^{*}:=} & \mathbb{E}_{t_{N-n+1}} \\
& \times\left[\exp \left(-\gamma_{t_{N-n}}\left(\sum_{k=N-n+1}^{N-1} \Delta X_{t_{k}}^{*}+I_{t_{N}}\right)\right)\right], \\
e^{-\gamma_{t_{0}} \widehat{Y}_{t_{N-n+1}}:=} & \mathbb{E}_{t_{N-n+1}} \\
& \times\left[\exp \left(-\gamma_{t_{0}}\left(\sum_{k=N-n+1}^{N-1} \Delta \widehat{X}_{t_{k}}+I_{t_{N}}\right)\right)\right] .
\end{aligned}
$$

Here

$$
\Delta X_{t_{k}}^{*}=\alpha_{t_{k}}^{*}\left(\mu_{t_{k}}^{c} h+\sigma_{t_{k}}^{c} \sqrt{h} \Delta b_{t_{k}}^{1}\right)+m_{D} \Delta D_{t_{k}},
$$

for any $k=N-n+1, \ldots, N-2, N-1$; the subgame perfect strategy $\alpha_{t_{k}}^{*}$ is given by

$$
\begin{aligned}
\alpha_{t_{k}}^{*}= & \frac{1}{2 \gamma_{t_{k}} \sigma_{t_{k}}^{c} \sqrt{h}} \log \left(\frac{1+r_{t_{k}}^{c} \sqrt{h}}{1-r_{t_{k}}^{c} \sqrt{h}}\right) \\
& +\frac{1}{2 \gamma_{t_{k}} \sigma_{t_{k}}^{c} \sqrt{h}} \log \left(\frac{\mathbb{E}_{t_{k}}\left[e^{-\gamma_{t_{k}}\left(m_{D} D_{t_{k+1}}+Y_{t_{k+1}}^{*}\right)} \mid A_{t_{k}}\right]}{\mathbb{E}_{t_{k}}\left[e^{-\gamma_{t_{k}}\left(m_{D} D_{t_{k+1}}+Y_{t_{k+1}}^{*}\right)} \mid A_{t_{k}}^{c}\right]}\right) .
\end{aligned}
$$


Moreover

$$
\Delta \widehat{X}_{t_{k}}=\widehat{\alpha}_{t_{k}}\left(\mu_{t_{k}}^{c} h+\sigma_{t_{k}}^{c} \sqrt{h} \Delta b_{t_{k}}^{1}\right)+m_{D} \Delta D_{t_{k}},
$$

for any $k=N-n+1, \ldots, N-2, N-1$; the naive strategy $\widehat{\alpha}_{t_{k}}$ is given by

$$
\begin{aligned}
\widehat{\alpha}_{t_{k}}= & \frac{1}{2 \gamma_{t_{0}} \sigma_{t_{k}}^{c} \sqrt{h}} \log \left(\frac{1+r_{t_{k}}^{c} \sqrt{h}}{1-r_{t_{k}}^{c} \sqrt{h}}\right) \\
& +\frac{1}{2 \gamma_{t_{0}} \sigma_{t_{k}}^{c} \sqrt{h}} \log \left(\frac{\mathbb{E}_{t_{k}}\left[e^{-\gamma_{t_{0}}\left(m_{D} D_{t_{k+1}}+\widehat{Y}_{t_{k+1}}\right)} \mid A_{t_{k}}\right]}{\mathbb{E}_{t_{k}}\left[e^{-\gamma_{t_{0}}\left(m_{D} D_{t_{k+1}}+\widehat{Y}_{t_{k+1}}\right)} \mid A_{t_{k}}^{c}\right]}\right) .
\end{aligned}
$$

Next, define the one-step period pricing kernels $\Lambda_{t_{N-n+1}}^{*}$ and $\widehat{\Lambda}_{t_{N-n+1}}$ by

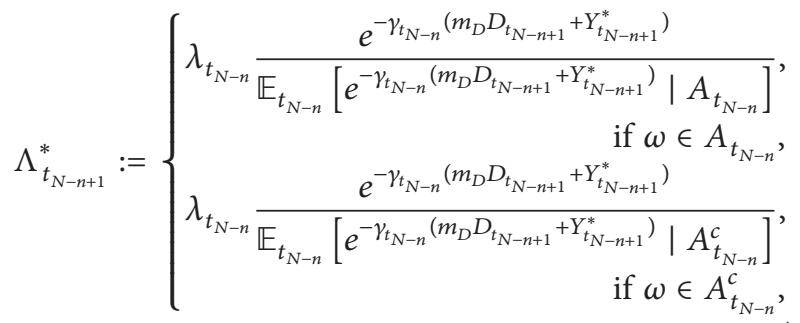

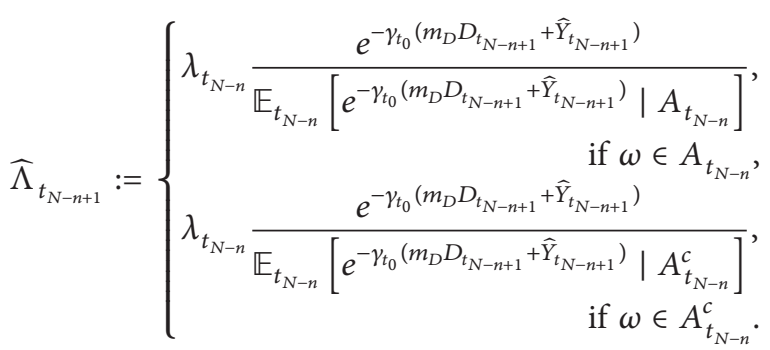

Here

$$
\lambda_{t_{N-n}}= \begin{cases}1-r_{t_{N-n}}^{c} \sqrt{h}, & \text { if } \omega \in A_{t_{N-n}} \\ 1+r_{t_{N-n}}^{c} \sqrt{h}, & \text { if } \omega \in A_{t_{N-n}^{c}}^{c}\end{cases}
$$

Remark 6. Notice that $\widehat{Y}_{t_{N-n+1}}$, defined by (30), is the certainty equivalent at time $t_{N-n+1}$ of the naive strategy which does not update the risk aversion and assumes constant risk aversion $\gamma_{t_{0}}$. Similarly, $Y_{t_{N-n+1}^{*}}^{*}$ can be seen as the certainty equivalent of the subgame perfect strategy with updating risk preference over time. In the special case of constant coefficient of absolute risk aversion, $\widehat{Y}_{t_{N-n+1}}$ and $Y_{t_{N-n+1}}^{*}$ are equal. Furthermore, it can be easily seen that

$$
\begin{aligned}
e^{-\gamma_{t_{0}} \widehat{Y}_{t_{N-n+1}}}= & \mathbb{E}_{t_{N-n+1}} \\
& \times\left[\exp \left(-\gamma_{t_{0}}\left(\Delta \widehat{X}_{t_{N}-n+1}+\widehat{Y}_{N-n+2}\right)\right)\right],
\end{aligned}
$$

where $\widehat{X}_{t_{N}-n+1}$ is the change of self-financed portfolio value during the time period $[(N-n+1) h,(N-n+2) h]$ so that
$\widehat{Y}_{t_{N-n+2}}$ can be used to find out the values of $\widehat{Y}_{t_{N-n+1}}$. However, we can observe that

$$
\begin{aligned}
e^{-\gamma_{t_{N-n}} Y_{t_{N-n+1}}^{*}} \neq & \mathbb{E}_{t_{N-n+1}} \\
& \times\left[\exp \left(-\gamma_{t_{N-n}}\left(\Delta X_{t_{N}-n+1}^{*}+Y_{N-n+2}^{*}\right)\right)\right]
\end{aligned}
$$

because the risk aversion changes over time in the definition of $Y^{*}$ in (29). More precisely, $Y_{t_{N-n+1}^{*}}^{*}$ is defined with $\gamma_{t_{N-n}}$, while $Y_{t_{N-n+2}}^{*}$ is defined with $\gamma_{t_{N-n+1}}$ which can be different from $\gamma_{t_{N-n}}$. Therefore, all possible future changes of risk aversion until the time horizon $T$ should be considered to compute the value of $Y_{N-n+1}^{*}$. Moreover, the pricing kernel $\Lambda_{t_{N-n+1}}^{*}$ in (35) for subgame perfect equilibrium price contains $Y_{t_{N-n+1}^{*}}^{*}$ as well as $\gamma_{t_{N-n}}$. As a consequence, all possible future changes of risk aversion should be examined to find the pricing kernel $\Lambda_{t_{N-n+1}^{*}}^{*}$.

Remark 7. The myopic term in $\alpha_{t_{k}}^{*}$ in (32) is $\left(1 / 2 \gamma_{t_{k}} \sigma_{t_{k}}^{c} \sqrt{h}\right)$ $\log \left(\left(1+r_{t_{k}}^{c} \sqrt{h}\right) /\left(1-r_{t_{k}}^{c} \sqrt{h}\right)\right)$ and in $\widehat{\alpha}_{t_{k}}$ is $\left(1 / 2 \gamma_{t_{0}} \sigma_{t_{k}}^{c} \sqrt{h}\right) \log ((1+$ $\left.\left.r_{t_{k}}^{c} \sqrt{h}\right) /\left(1-r_{t_{k}}^{c} \sqrt{h}\right)\right)$. Moreover, it can be verified that they satisfy

$$
\begin{aligned}
& \lim _{h \downarrow 0} \frac{1}{2 \gamma_{t_{k}} \sigma_{t_{k}}^{c} \sqrt{h}} \log \left(\frac{1+r_{t_{k}}^{c} \sqrt{h}}{1-r_{t_{k}}^{c} \sqrt{h}}\right)=\frac{r_{t_{k}}^{c}}{\gamma_{t_{k}} \sigma_{t_{k}}^{c}}, \\
& \lim _{h \downarrow 0} \frac{1}{2 \gamma_{t_{0}} \sigma_{t_{k}}^{c} \sqrt{h}} \log \left(\frac{1+r_{t_{k}}^{c} \sqrt{h}}{1-r_{t_{k}}^{c} \sqrt{h}}\right)=\frac{r_{t_{k}}^{c}}{\gamma_{t_{0}} \sigma_{t_{k}}^{c}},
\end{aligned}
$$

respectively. Notice that the right-hand sides of (41) are the well-known myopic term of the optimal investment strategy with constant absolute risk aversion $\gamma_{t_{0}}$ in continuous time model. The only difference in (40) with subgame perfect strategy is that the risk aversion is updated; that is, $\gamma_{t_{k}}$ is used, while it is not in the naive strategy (41). The second terms of (32) and (34) are hedging terms which take into account the other factors, such as investment in derivative, stochastic parameters of underlying processes, income, and stochastic risk aversion (in case of subgame perfect strategy).

The following theorem is the main result of the paper.

Theorem 8. The subgame perfect equilibrium price at time $t_{N-n}$ is given by

$$
D_{t_{N-n}}=\mathbb{E}_{t_{N-n}}^{\mathbb{Q}^{*}}\left[D_{t_{N-n+1}}\right],
$$

where the probability measure $\mathbb{Q}^{*}$ is defined by

$$
\frac{d \mathbb{Q}^{*}}{d \mathbb{P}}=\Lambda_{t_{N}}^{*} \Lambda_{t_{N-1}}^{*} \cdots \Lambda_{t_{1}}^{*}
$$

The subgame perfect strategy (in the primary asset) is $\alpha^{*}=$ $\left(\alpha_{t_{0}}^{*}, \alpha_{t_{1}}^{*}, \ldots, \alpha_{t_{N}}^{*}\right)$, with $\alpha_{t_{k}}^{*}$ defined by (32). The naive equilibrium price at time $t_{N-n}$ is given by

$$
D_{t_{N-n}}=\mathbb{E}_{t_{N-n}}^{\widehat{\mathbb{Q}}}\left[D_{t_{N-n+1}}\right] \text {, }
$$


where the probability measure $\widehat{\mathbb{Q}}$ is defined by

$$
\frac{d \widehat{\mathbb{Q}}}{d \mathbb{P}}=\widehat{\Lambda}_{t_{N}} \widehat{\Lambda}_{t_{N-1}} \cdots \widehat{\Lambda}_{t_{1}} .
$$

The naive strategy (in the primary asset) is $\widehat{\alpha}=\left(\widehat{\alpha}_{t_{0}}, \widehat{\alpha}_{t_{1}}, \ldots\right.$, $\widehat{\alpha}_{t_{N}}$ ), with $\widehat{\alpha}_{t_{k}}$ defined by (34).

Proof. Proof of this theorem is done in Appendix B.

For the naive equilibrium price, we recover the following classical result.

Corollary 9. The pricing kernel for naive equilibrium price equals the marginal utility; that is,

$$
\widehat{\Lambda}_{t_{N-n+1}}=\frac{\mathbb{E}_{t_{N-n+1}}\left[U^{\prime}\left(\widehat{W}_{t_{N}}\right)\right]}{\mathbb{E}_{t_{N-n}}\left[U^{\prime}\left(\widehat{W}_{t_{N}}\right)\right]},
$$

where $U(x)=-e^{-\gamma x}$, and $\widehat{W}_{t_{N}}$ (see (5)) is the wealth obtained by naive strategies.

Proof. Proof of this corollary is done in Appendix C.

Lemma 10. The tradable assets $\left\{C_{t_{n}}\right\}_{n=0,1, \ldots, N}$ and $\left\{D_{t_{n}}\right\}_{n=0,1, \ldots, N}$ are martingales under $Q^{*}$.

Proof. It can be proved by following similar argument to the proof of Lemma 5.

\section{Numerical Results}

Throughout this section, we specialize to a regime switching model. A discrete time finite state homogeneous Markov chain $(\mathrm{MC}) J:=\left(J_{t_{n}}\right)_{n=0,1, \ldots, \infty}$ is defined on $\left(\Omega, \mathscr{F}_{,},\left\{\mathscr{F}_{t_{n}}\right\}, \mathbb{P}\right)$ and it takes values in the state space $\mathcal{S}=\{\mathbf{0}, \mathbf{1}\}$ (which represents two states of the market: bull and bear). The $n$-step transition matrix $P^{(n)}=\left(P_{i j}^{n}\right)$, is defined by

$$
P_{i j}^{(n)}:=\mathbb{P}\left(J_{t_{n}}=j \mid J_{t_{0}}=i\right), \quad i, j=\mathbf{0}, \mathbf{1}, n=0,1, \ldots, \infty,
$$

where $P_{i j}^{(0)}=1$ when $i=j$; otherwise $P_{i j}^{(0)}=0$. We assume that the distribution of $J_{0}$ is known, and

$$
\mathbb{P}\left(J_{0}=i \mid \mathscr{F}_{0}\right)=\mathbb{P}\left(J_{0}=i\right), \quad i=\mathbf{0}, \mathbf{1} .
$$

The risk aversion is changed depending on the state and denoted by $\gamma_{t_{n}}=\gamma\left(J_{t_{n}} \cdot\right)$

In this section we give a concrete example. Consider the electricity industry one of the most weather-sensitive businesses in the economy. Energy price and temperature are denoted by $C$ and $S$, respectively. When the temperature increases, there is a higher demand for electricity due to the usage of air conditioners. In turn this will lead to higher energy prices. Therefore, $C$ and $S$ are assumed to be positively correlated. In our model an energy provider hedges the weather exposure by selling $m_{D}$ shares of weather derivative to the representative agent. This derivative is designed to have a higher payoff when temperature is high (e.g., call option). It is supposed that the representative agent's income depends on the weather, so he/she has an incentive to buy this product because of his/her income exposure to the weather. Moreover, weather derivative also allows the representative agent to construct a more diversified portfolio. The baseline parameters are

$$
\begin{gathered}
C_{0}=1, \quad \mu^{c}=0.1, \quad \sigma^{c}=0.2, \quad S_{0}=1, \\
\mu^{s}=0.1, \quad \sigma^{s}=0.2, \quad \rho=0.5, \\
T=1, \quad N=52, \quad h=\frac{1}{52}, \quad \gamma(0)=1, \\
\gamma(1)=2, \quad m_{D}=1, \\
I_{t_{N}}=4 \exp \left(-0.1\left(S_{t_{N}}-S_{0}\right)\right), \\
D_{t_{N}}=\left(S_{t_{N}}-K\right)^{+}, \quad K=1 .
\end{gathered}
$$

One-step transition matrix is given by

$$
P^{(1)}=\left(\begin{array}{ll}
0.6 & 0.4 \\
0.6 & 0.4
\end{array}\right) \text {. }
$$

Notice that $S$ is the normalized temperature. The income of the agent is assumed to be exponential function of $S$ to prevent negative income. However, our result in this section still can be obtained if we use different models for income (e.g., linear function of $S$ ). Since the income is decreasing in $S$, the agent has an incentive to have long position in $S$ which is impossible because $S$ is a nontradable asset. Instead, there is a demand for $D$ which is a call option on nontradable asset $S$. Letting $N=52$ with $T=1$ means weekly rebalancing of portfolio throughout a year. In fact, equilibrium price changes if we consider large $N$. However, the change of price is very small (less than $0.2 \%$ even if we consider $N=200$ ) so we may only focus on the results with $N=52$.

Let $D_{0}^{\gamma(0), \gamma(1)}$ be the subgame perfect equilibrium price of $D$ at time 0 with $J_{0}=0$ and time changing risk aversion. Likewise, $D_{0}^{\gamma(1), \gamma(0)}$ is the subgame perfect equilibrium price of $D$ at time 0 with $J_{0}=1$. It is natural to define $D_{0}^{\gamma, \gamma}$ as the naive prices of $D$ at time 0 with risk aversion $\gamma$. Recall that this naive price ignores the time changing risk aversion of the agent.

4.1. Effect of Risk Aversion. First of all, let us consider the effect of risk aversion on the naive equilibrium price. As we can see in Figure 1, the naive equilibrium price $D_{0}^{\gamma, \gamma}$ decreases as risk aversion $\gamma$ increases. This is intuitive because the derivative $D$ is also a risky asset and there is a less demand for risky asset if the agent is more risk averse. A lower demand for $D$ leads to a lower naive equilibrium price as a result. In sum, a higher risk aversion results in a lower naive equilibrium price.

Therefore, if we consider the naive prices $D_{0}^{\gamma(0), \gamma(0)}$ and $D_{0}^{\gamma(1), \gamma(1)}$ for different risk aversions $\gamma(0)$ and $\gamma(1)$ which satisfy $\gamma(0)<\gamma(1)$, we have

$$
D_{0}^{\gamma(0), \gamma(0)}>D_{0}^{\gamma(1), \gamma(1)} .
$$




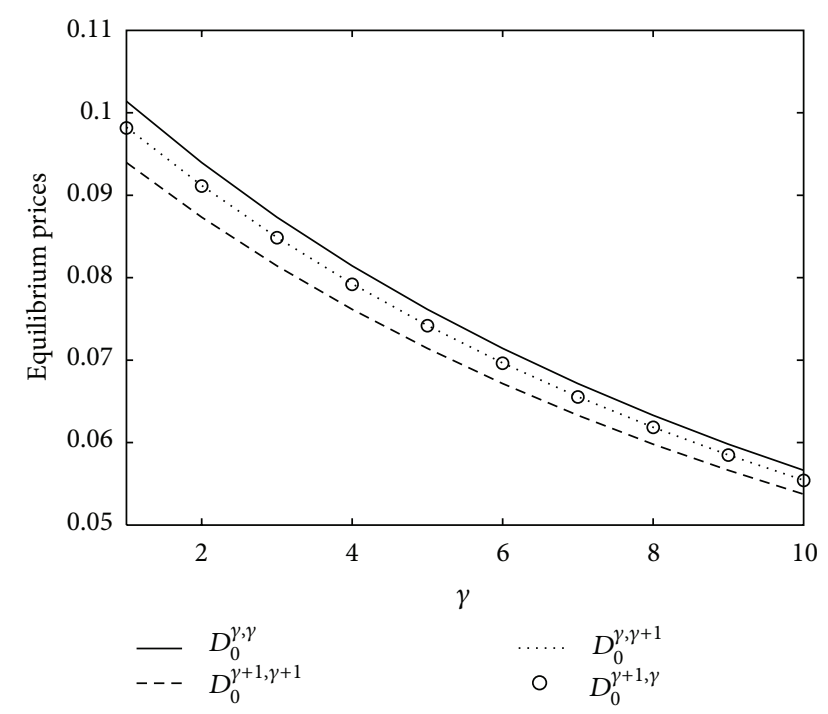

FIGURE 1: Effect of risk aversion on the equilibrium prices.

From (51), we can infer that if $\gamma(0)<\gamma(1)$, the subgame perfect equilibrium prices $D_{0}^{\gamma(0), \gamma(1)}$ and $D_{0}^{\gamma(1), \gamma(0)}$ are in between $D_{0}^{\gamma(0), \gamma(0)}$ and $D_{0}^{\gamma(1), \gamma(1)}$; that is,

$$
\begin{aligned}
& D_{0}^{\gamma(1), \gamma(1)}<D_{0}^{\gamma(0), \gamma(1)}<D_{0}^{\gamma(0), \gamma(0)}, \\
& D_{0}^{\gamma(1), \gamma(1)}<D_{0}^{\gamma(1), \gamma(0)}<D_{0}^{\gamma(0), \gamma(0)} .
\end{aligned}
$$

In Figure 1, we can verify the inequalities in (52).

Let $\gamma_{p}$ be the weighted average of $\gamma(0)$ and $\gamma(1)$ with steady-state probabilities $P_{0}$ and $P_{1}$ of states $\mathbf{0}$ and $\mathbf{1}$ as weights; that is,

$$
\gamma_{p}:=P_{0} \gamma(0)+P_{1} \gamma(1)
$$

Consider the naive equilibrium price $D_{0}^{\gamma_{p}, \gamma_{p}}$ with $\gamma_{p}$, and then a question arises. Is the naive equilibrium price $D_{0}^{\gamma_{p}, \gamma_{p}}$ a good estimate of the subgame perfect equilibrium prices $D_{0}^{\gamma(0), \gamma(1)}$ and $D_{0}^{\gamma(1), \gamma(0)}$ ? If the gap between $D_{0}^{\gamma_{p}, \gamma_{p}}$ and the subgame perfect equilibrium prices is small enough, we may use the naive equilibrium price $D_{0}^{\gamma_{p}, \gamma_{p}}$ instead of the subgame perfect equilibrium prices. However, the answer is no because the gap is considerable for many cases.

In Figure 2, $\gamma(0)$ is fixed at $\gamma(0)=1$ and we examine the changes of equilibrium prices as $\gamma(1)$ changes. The left panel shows the equilibrium prices; the solid line is $D_{0}^{\gamma_{p}, \gamma_{p}}$, the dashed line is $D_{0}^{\gamma(0), \gamma(1)}$, and the dotted line is $D_{0}^{\gamma(1), \gamma(0)}$. In the right panel, percentage changes from $D_{0}^{\gamma_{p}, \gamma_{p}}$ are illustrated; that is,

$$
\begin{aligned}
& \text { Dashed line: } \frac{D_{0}^{\gamma(0), \gamma(1)}-D_{0}^{\gamma_{p}, \gamma_{p}}}{D_{0}^{\gamma_{p}, \gamma_{p}}} \times 100 \\
& \text { Dotted line: } \frac{D_{0}^{\gamma(1), \gamma(0)}-D_{0}^{\gamma_{p}, \gamma_{p}}}{D_{0}^{\gamma_{p}, \gamma_{p}}} \times 100
\end{aligned}
$$

We can observe that there are significant gaps between the naive equilibrium price $D_{0}^{\gamma_{p}, \gamma_{p}}$ and the subgame perfect equilibrium prices $D_{0}^{\gamma(0), \gamma(1)}$ and $D_{0}^{\gamma(1), \gamma(0)}$ for large enough $\gamma(1)$, and the gaps become wider as $\gamma(1)$ increases. This implies that if the agent becomes much more risk averse in the bear market than in the bull market, the naive equilibrium price with $\gamma_{p}$ cannot be a good estimate of the subgame perfect equilibrium prices and thus the subgame perfect equilibrium prices should be derived using our pricing principle.

4.2. Effect of $m_{D}$. Now we examine the effect of $m_{D}$, the number of units of derivative $D$ in the market, on the equilibrium prices.

It is observed that the equilibrium prices (both subgame perfect and naive) are decreasing with respect to $m_{D}$ in the left panel of Figure 3. This is a consistent result with Gârleanu et al. [25]. They considered a derivative market with two participants: representative dealer and end users. End users' aggregate demand for derivative $d$ is assumed to be a random variable. On the contrary, dealer's demand for derivative $q$ is optimally determined by utility maximization. The market clearing condition is $q+d=0$. Their result is

$$
\frac{\partial p}{\partial d} \geq 0
$$

Notice that as $d$ decreases, there are more derivatives which should be purchased by representative dealer to achieve market clearing. So the decreasing $d$ in Gârleanu et al. [25] corresponds to increasing $m_{D}$ in our model. Therefore, our result

$$
\frac{\partial D_{0}}{\partial m_{D}}<0
$$

is consistent with (55). Moreover, as we can observe in the right panel of Figure 3, the gaps between the subgame perfect equilibrium prices $D_{0}^{\gamma(0), \gamma(1)}$ and $D_{0}^{\gamma(1), \gamma(0)}$ and the naive equilibrium price $D_{0}^{\gamma_{p}, \gamma_{p}}$ is considerable for large enough $m_{D}$.

\section{Conclusion}

We consider a multiperiod incomplete market model with tradable risky asset, nontradable underlying and contingent claim (derivative) written on both tradable risky asset, and nontradable underlying. We develop an equilibrium pricing principle for the derivative; the price of the derivative is priced by combining the optimal strategies of representative agent and market clearing condition for the derivative. Since the agent's risk aversion is assumed to be stochastic, classical approach with precommitment gives the naive strategies and the naive equilibrium price which do not update the change of risk preference. Alternatively, we consider the subgame perfect strategies and derive the corresponding subgame perfect equilibrium price. We consider two-state regime switching model for numerical experiments. Then the numerical result shows that naive equilibrium price cannot be a good estimate for subgame perfect equilibrium price in many cases. In particular, if the representative agent's risk 


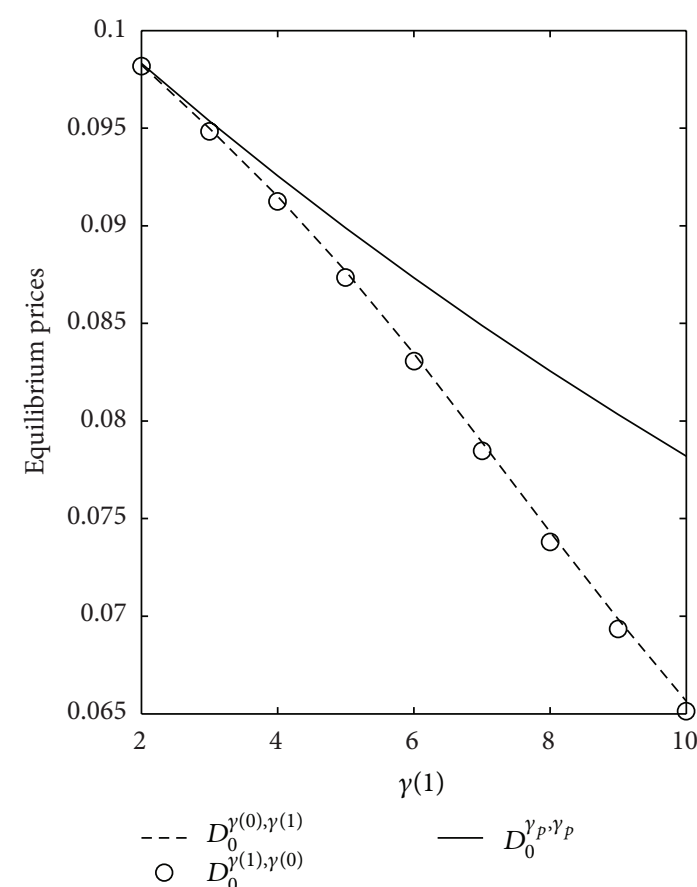

(a)

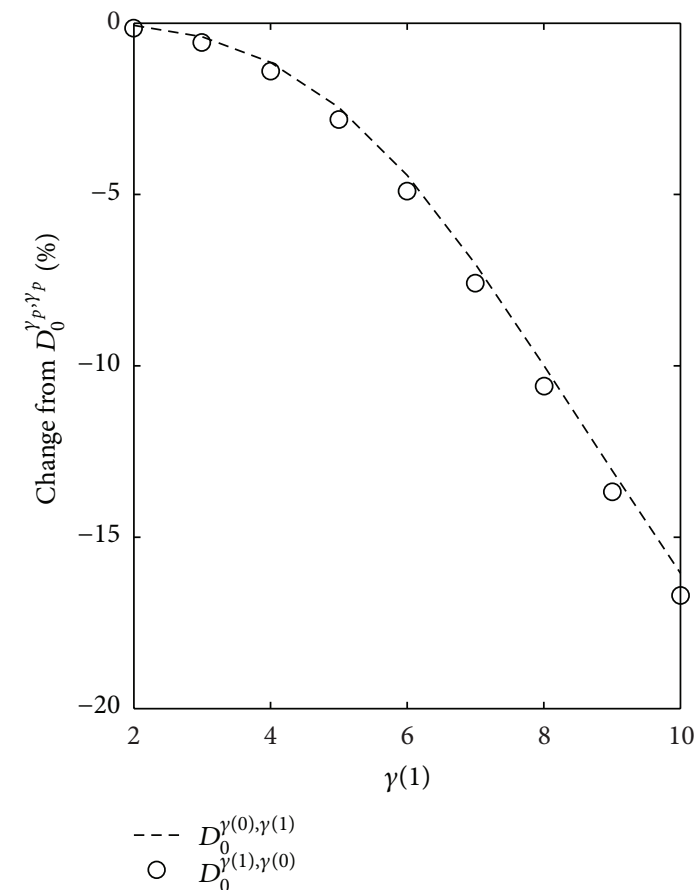

(b)

FIgURE 2: The gap between $D_{0}^{\gamma_{p}, \gamma_{p}}$ and the subgame perfect equilibrium prices. $\gamma(0)$ is fixed at 1 in both panels.

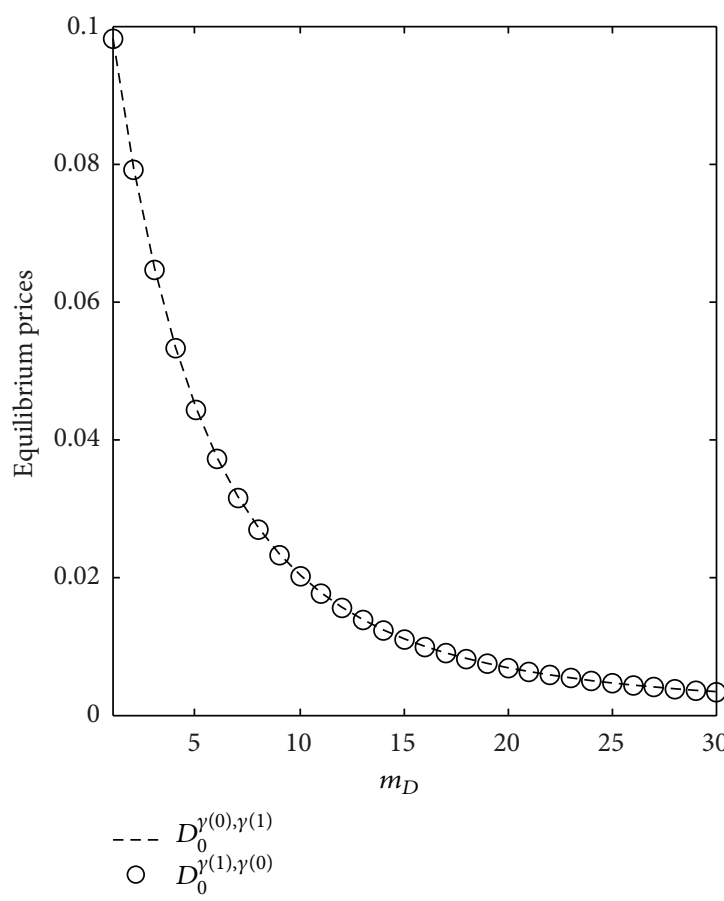

(a)

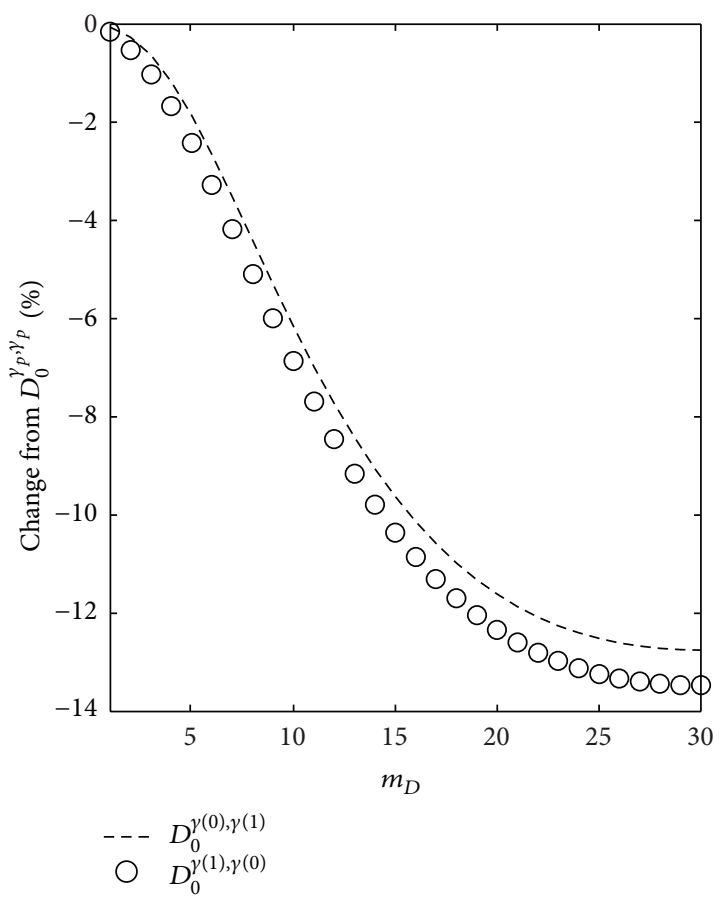

(b)

FIGURE 3: The gap between $D_{0}^{\gamma_{p}, \gamma_{p}}$ and the subgame perfect equilibrium prices. 
aversion changes a lot depending on regime or the number of units of derivative we consider is large, then the gap between naive equilibrium price and subgame perfect equilibrium price is considerable. This shows the importance of our subgame perfect equilibrium pricing principle.

\section{Appendices}

\section{A. Proof of Theorem 3}

From (4) and (5), it follows that

$$
\begin{aligned}
\mathbb{E}_{t_{N-1}} & {\left[-\exp \left(-\gamma_{t_{N-1}}\left(X_{t_{N}}^{\pi}+I_{t_{N}}\right)\right)\right] } \\
& =\mathbb{E}_{t_{N-1}}\left[-\exp \left(-\gamma_{t_{N-1}}\left(X_{t_{N-1}}+\Delta X_{t_{N-1}}^{\pi}+I_{t_{N}}\right)\right)\right] \\
& =-e^{-\gamma_{t_{N-1}} X_{t_{N-1}}^{\pi}} \mathbb{E}_{t_{N-1}}\left[\exp \left(-\gamma_{t_{N-1}}\left(\Delta X_{t_{N-1}}^{\pi}+I_{t_{N}}\right)\right)\right] \\
& =-e^{-\gamma_{t_{N-1}} X_{t_{N-1}}^{\pi}} g_{N-1}(\alpha, \beta, \cdot),
\end{aligned}
$$

where

$$
\begin{aligned}
g_{N-1}(\alpha, \beta,):= & \mathbb{E}_{t_{N-1}}\left[\exp \left(-\gamma_{t_{N-1}}\left(\Delta X_{t_{N-1}}^{\pi}+I_{t_{N}}\right)\right)\right] \\
= & \frac{1}{2} e^{-\gamma_{t_{N-1}} \alpha\left(\mu_{t_{N-1}}^{c} h+\sigma_{t_{N-1}}^{c} \sqrt{h}\right)} \\
& \times \mathbb{E}_{t_{N-1}}\left[e^{-\gamma_{t_{N-1}} \beta \Delta D_{t_{N-1}}} e^{-\gamma_{t_{N-1}} I_{t_{N}}} \mid A_{t_{N-1}}\right] \\
& +\frac{1}{2} e^{-\gamma_{t_{N-1}} \alpha\left(\mu_{t_{N-1}}^{c} h-\sigma_{t_{N-1}}^{c} \sqrt{h}\right)} \\
& \times \mathbb{E}_{t_{N-1}}\left[e^{-\gamma_{t_{N-1}} \beta \Delta D_{t_{N-1}}} e^{-\gamma_{t_{N-1}} I_{t_{N}}} \mid A_{t_{N-1}}^{c}\right] .
\end{aligned}
$$

Recall that $A_{t_{k}}:=\left\{\omega \in \Omega: \Delta b_{t_{k}}^{1}=1\right\}$ and $A_{t_{k}}^{c}:=\{\omega \in$ $\left.\Omega: \Delta b_{t_{k}}^{1}=-1\right\}$. The function $g_{N-1}(\alpha, \beta, \cdot)$ has the following properties:

$$
g_{N-1}(0,0, \cdot)=\mathbb{E}_{t_{N-1}}\left[e^{-\gamma_{t_{N-1}} I_{t_{N}}}\right] \leq 1 .
$$

For a fixed $\beta$, it follows that for small $h$

$$
g_{N-1}(\infty, \beta, \cdot)=\infty ; \quad g_{N-1}(-\infty, \beta, \cdot)=\infty
$$

By arbitrage argument it follows that $D_{t_{N-1}}$ belongs to the interval

$$
D_{t_{N-1}}(\omega) \in\left[\inf _{\mathbb{Q}} \mathbb{E}^{\mathbb{Q}}\left[D_{t_{N}}\right], \sup _{\mathbb{Q}} \mathbb{E}^{\mathbb{Q}}\left[D_{t_{N}}\right]\right], \quad \omega \in \Omega,
$$

where $\mathbb{Q}$ ranges over the set of probability measures. Consequently,

$$
\Delta D_{t_{N-1}}(\omega) \in\left[D_{t_{N}}-\sup _{\mathbb{Q}} \mathbb{E}^{\mathbb{Q}}\left[D_{t_{N}}\right], D_{t_{N}}-\inf _{\mathbb{Q}} \mathbb{E}^{\mathbb{Q}}\left[D_{t_{N}}\right]\right] .
$$

Thus, the sets $\left\{\omega \in \Omega: \Delta D_{t_{N-1}}(\omega)>0\right\}$ and $\{\omega \in \Omega$ : $\left.\Delta D_{t_{N-1}}(\omega)<0\right\}$ have positive probability. This implies that

$$
g_{N-1}(\alpha, \infty, \cdot)=\infty, \quad g_{N-1}(\alpha,-\infty, \cdot)=\infty
$$

From the above analysis, it follows that the minimum of the function of $g$ is a critical point. First order conditions lead to

$$
\begin{aligned}
& \frac{\partial g_{N-1}}{\partial \alpha}=\frac{-\gamma_{t_{N-1}}\left(\mu_{t_{N-1}}^{c} h+\sigma_{t_{N-1}}^{c} \sqrt{h}\right)}{2} \\
& \cdot e^{-\gamma_{t_{N-1}} \alpha\left(\mu_{t_{N-1}}^{c} h+\sigma_{t_{N-1}}^{c} \sqrt{h}\right)} \\
& \times \mathbb{E}_{t_{N-1}}\left[e^{-\gamma_{t_{N-1}} \beta \Delta D_{t_{N-1}}} e^{-\gamma_{t_{N-1}} I_{t_{N}}} \mid A_{t_{N-1}}\right] \\
& +\frac{-\gamma_{t_{N-1}}\left(\mu_{t_{N-1}}^{c} h-\sigma_{t_{N-1}}^{c} \sqrt{h}\right)}{2} \\
& \cdot e^{-\gamma_{t_{N-1}} \alpha\left(\mu_{t_{N-1}}^{c} h-\sigma_{t_{N-1}}^{c} \sqrt{h}\right)} \\
& \times \mathbb{E}_{t_{N-1}}\left[e^{-\gamma_{t_{N-1}} \beta \Delta D_{t_{N-1}}} e^{-\gamma_{t_{N-1}} I_{t_{N}}} \mid A_{t_{N-1}}^{c}\right] \\
& =0 \text {, } \\
& \frac{\partial g_{N-1}}{\partial \beta}=\frac{1}{2} e^{-\gamma_{t_{N-1}} \alpha\left(\mu_{t_{N-1}}^{c} h+\sigma_{t_{N-1}}^{c} \sqrt{h}\right)} \\
& \times \mathbb{E}_{t_{N-1}}\left[-\gamma_{t_{N-1}} \Delta D_{t_{N-1}} e^{-\gamma_{t_{N-1}} \beta \Delta D_{t_{N-1}}}\right. \\
& \left.\times e^{-\gamma_{t_{N-1}} I_{t_{N}}} \mid A_{t_{N-1}}\right] \\
& \left.+\frac{1}{2} e^{-\gamma_{t_{N-1}} \alpha\left(\mu_{t_{N-1}}^{c} h-\sigma_{t_{N-1}}^{c}\right.} \sqrt{h}\right) \\
& \times \mathbb{E}_{t_{N-1}}\left[-\gamma_{t_{N-1}} \Delta D_{t_{N-1}} e^{-\gamma_{t_{N-1}} \beta \Delta D_{t_{N-1}}}\right. \\
& \left.\times e^{-\gamma_{t_{N-1}} I_{t_{N}}} \mid A_{t_{N-1}}^{c}\right]
\end{aligned}
$$

$=0$.

Recall that

$$
\Delta D_{t_{N-1}}=D_{t_{N}}-\mathbb{E}_{t_{N-1}}^{\mathbb{Q}^{*}}\left[D_{t_{N}}\right]
$$

for an equilibrium pricing measure $\mathbb{Q}^{*}$ to be found. Since $\mathbb{E}_{t_{N-1}}^{\mathbb{Q}^{*}}\left[D_{t_{N}}\right]$ is $\mathscr{F}_{t_{N-1}}$ measurable, it follows that

$$
\begin{aligned}
\alpha_{t_{N-1}}^{*}= & \frac{1}{2 \gamma_{t_{N-1}} \sigma_{t_{N-1}}^{c} \sqrt{h}} \log \left(\frac{1+r_{t_{N-1}}^{c} \sqrt{h}}{1-r_{t_{N-1}}^{c} \sqrt{h}}\right) \\
& +\frac{1}{2 \gamma_{t_{N-1}} \sigma_{t_{N-1}}^{c} \sqrt{h}} \\
& \times \log \left(\frac{\mathbb{E}_{t_{N-1}}\left[e^{\left.-\gamma_{t_{N-1}} \beta_{t_{N-1}}^{*} D_{t_{N}} e^{-\gamma_{t_{N-1}} I_{t_{N}}} \mid A_{t_{N-1}}\right]}\right.}{\mathbb{E}_{t_{N-1}}\left[e^{-\gamma_{t_{N-1}} \beta_{t_{N-1}}^{*} D_{N}} e^{-\gamma_{t_{N-1}} I_{t_{N}}} \mid A_{t_{N-1}}^{c}\right]}\right) .
\end{aligned}
$$


By the equilibrium condition $\beta_{t_{N-1}}^{*}=m_{D}$. This together with $\partial g_{N-1} / \partial \beta=0$ leads to

$$
\begin{aligned}
& \mathbb{E}_{t_{N-1}}\left[\Delta D_{t_{N-1}} e^{-\gamma_{t_{N-1}} m_{D} \Delta D_{t_{N-1}}} e^{-\gamma_{t_{N-1}} I_{t_{N}}} \mid A_{t_{N-1}}\right] \\
& =-e^{2 \gamma_{t_{N-1}} \alpha_{t_{N-1}}^{*} \sigma_{t_{N-1}}^{c} \sqrt{h}} \mathbb{E}_{t_{N-1}} \\
& \times\left[\Delta D_{t_{N-1}} e^{-\gamma_{t_{N-1}} m_{D} \Delta D_{t_{N-1}}} e^{-\gamma_{t_{N-1}} I_{t_{N}}} \mid A_{t_{N-1}}^{c}\right], \\
& e^{2 \gamma_{t_{N-1}} \alpha_{t_{N-1}}^{*} \sigma_{t_{N-1}}^{c} \sqrt{h}} \\
& =\frac{\left(1+r_{t_{N-1}}^{c} \sqrt{h}\right) \mathbb{E}_{t_{N-1}}\left[e^{-\gamma_{t_{N-1}} m_{D} D_{t_{N}}} e^{-\gamma_{t_{N-1}} I_{t_{N}}} \mid A_{t_{N-1}}\right]}{\left(1-r_{t_{N-1}}^{c} \sqrt{h}\right) \mathbb{E}_{t_{N-1}}\left[e^{-\gamma_{t_{N-1}} m_{D} D_{t_{N}}} e^{-\gamma_{t_{N-1}} I_{t_{N}}} \mid A_{t_{N-1}}^{c}\right]} .
\end{aligned}
$$

Combing the above equations leads to

$$
\begin{aligned}
& \frac{2}{1-r_{t_{N-1}}^{c} \sqrt{h}} \mathbb{E}_{t_{N-1}}^{\mathbb{Q}^{*}}\left[D_{t_{N}}\right] \\
& =\frac{\mathbb{E}_{t_{N-1}}\left[D_{t_{N}} e^{-\gamma_{t_{N-1}} m_{D} D_{t_{N}}} e^{-\gamma_{t_{N-1}} I_{t_{N}}} \mid A_{t_{N-1}}\right]}{\mathbb{E}_{t_{N-1}}\left[e^{-\gamma_{t_{N-1}} m_{D} D_{t_{N}}} e^{-\gamma_{t_{N-1}} I_{t_{N}}} \mid A_{t_{N-1}}\right]} \\
& +\frac{\left(1+r_{t_{N-1}^{c}}^{c} \sqrt{h}\right)}{\left(1-r_{t_{N-1}}^{c} \sqrt{h}\right)} \\
& \times \frac{\mathbb{E}_{t_{N-1}}\left[D_{t_{N}} e^{-\gamma_{t_{N-1}} m_{D} D_{t_{N}}} e^{-\gamma_{t_{N-1}} I_{t_{N}}} \mid A_{t_{N-1}}^{c}\right]}{\mathbb{E}_{t_{N-1}}\left[e^{-\gamma_{t_{N-1}} m_{D} D_{t_{N}}} e^{-\gamma_{t_{N-1}} I_{t_{N}}} \mid A_{t_{N-1}}^{c}\right]} .
\end{aligned}
$$

This together with (A.9) implies that

$$
\begin{aligned}
D_{t_{N-1}}= & \frac{1-r_{t_{N-1}}^{c} \sqrt{h}}{2} \\
& \times \frac{\mathbb{E}_{t_{N-1}}\left[D_{t_{N}} e^{-\gamma_{t_{N-1}} m_{D} D_{t_{N}}} e^{-\gamma_{t_{N-1}} I_{t_{N}}} \mid A_{t_{N-1}}\right]}{\mathbb{E}_{t_{N-1}}\left[e^{-\gamma_{t_{N-1}} m_{D} D_{t_{N}}} e^{-\gamma_{t_{N-1}} I_{t_{N}}} \mid A_{t_{N-1}}\right]} \\
& +\frac{1+r_{t_{N-1}}^{c} \sqrt{h}}{2} \\
& \times \frac{\mathbb{E}_{t_{N-1}}\left[D_{t_{N}} e^{-\gamma_{t_{N-1}} m_{D} D_{t_{N}}} e^{-\gamma_{t_{N-1}} I_{t_{N}}} \mid A_{t_{N-1}}^{c}\right]}{\mathbb{E}_{t_{N-1}}\left[e^{-\gamma_{t_{N-1}} m_{D} D_{t_{N}}} e^{-\gamma_{t_{N-1}} I_{t_{N}}} \mid A_{t_{N-1}}^{c}\right]} .
\end{aligned}
$$

Thus, the equilibrium price is

$$
D_{t_{N-1}}=\mathbb{E}_{t_{N-1}}\left[D_{t_{N}} \Lambda_{t_{N}}^{*}\right]
$$

where $\Lambda_{t_{N}}^{*}$ was defined in (22).

\section{B. Proof of Theorem 8}

We will prove the result for the subgame perfect equilibrium price; the proof for the naive equilibrium price is similar and hence omitted. First consider the time period $[(N-n) h,(N-$ $n+1) h)$ and choose an arbitrary control $\pi=(\alpha, \beta)$ as follows:

$$
\pi= \begin{cases}\pi_{t_{n}}^{*}, & \text { for } n=N-(n-1), N-(n-2), \ldots, N-1, \\ \pi_{t_{n}}, & \text { for } n=N-n\end{cases}
$$

From

$$
X_{t_{N}}^{\pi}=X_{t_{N-(n-1)}}^{\pi}+\sum_{k=N-(n-1)}^{N-1} \Delta X_{t_{k}}^{*}
$$

it follows that

$$
\begin{aligned}
& \mathbb{E}_{t_{N-n}}\left[-\exp \left(-\gamma_{t_{N-n}}\left(X_{t_{N}}^{\pi}+I_{t_{N}}\right)\right)\right] \\
& =-e^{-\gamma_{t_{N-n}} X_{t_{N-n}}^{\pi}} \mathbb{E}_{t_{N-n}} \\
& \times\left[e^{-\gamma_{t_{N-n}} \Delta X_{t_{N-n}}^{\pi}} \mathbb{E}_{t_{N-n+1}}\right. \\
& \left.\times\left[\exp \left(-\gamma_{t_{N-n}}\left(\sum_{k=N-(n-1)}^{N-1} \Delta X_{t_{k}}^{*}+I_{t_{N}}\right)\right)\right]\right] \\
& =-e^{-\gamma_{t_{N-n}} X_{t_{N-n}}^{\pi}} \mathbb{E}_{t_{N-n}}\left[e^{-\gamma_{t_{N-n}} \Delta X_{t_{N-n}}^{\pi}} e^{-\gamma_{t_{N-n}} Y_{t_{N-(n-1)}}^{*}}\right] \\
& =-e^{-\gamma_{t_{N-n}} X_{t_{N-n}}^{\pi}} g_{N-n}(\alpha, \beta, \cdot) \text {. }
\end{aligned}
$$

Here for $k=N-(n-1), \ldots, N-2, N-1$,

$$
\begin{aligned}
& \Delta X_{t_{k}}^{*}=\alpha_{t_{k}}^{*}\left(\mu_{t_{k}}^{c} h+\sigma_{t_{k}}^{c} \sqrt{h} \Delta b_{t_{k}}^{1}\right)+m_{D} \Delta D_{t_{k}},
\end{aligned}
$$

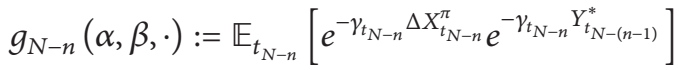

$$
\begin{aligned}
& =\frac{1}{2} e^{-\gamma_{t_{N-n}} \alpha\left(\mu_{t_{N-n}}^{c} h+\sigma_{t_{N-n}}^{c} \sqrt{h}\right)} \mathbb{E}_{t_{N-n}} \\
& \times\left[e^{-\gamma_{t_{N-n}} \beta \Delta D_{t_{N-n}}} e^{-\gamma_{t_{N-n}} Y_{t_{N-n+1}}^{*}} \mid A_{t_{N-n}}\right] \\
& +\frac{1}{2} e^{-\gamma_{t_{N-n}} \alpha\left(\mu_{t_{N-n}}^{c} h-\sigma_{t_{N-n}}^{c} \sqrt{h}\right)} \mathbb{E}_{t_{N-n}} \\
& \times\left[e^{-\gamma_{t_{N-n}} \beta \Delta D_{t_{N-n}}} e^{-\gamma_{t_{N-n}} Y_{t_{N-n+1}}^{*}} \mid A_{t_{N-n}}^{c}\right]
\end{aligned}
$$

with $A_{t_{N-n}}:=\left\{\omega \in \Omega: \Delta b_{t_{N-n}}^{1}=1\right\}$ and $A_{t_{N-n}}^{c}:=\{\omega \in \Omega$ : $\left.\Delta b_{t_{N-n}}^{1}=-1\right\}$. Arguing as in the one period case we get

$$
\begin{gathered}
g_{N-n}(0,0, \cdot)=\mathbb{E}_{t_{N-n}}\left[e^{-\gamma_{t_{N-n}} Y_{t_{N-n+1}}^{*}}\right] \leq \infty ; \\
g_{N-n}(\infty, \beta, \cdot)=\infty ; \quad g_{N-n}(-\infty, \beta, \cdot)=\infty .
\end{gathered}
$$

From arbitrage considerations it follows that

$$
D_{t_{N-n}}(\omega) \in\left[\inf _{\mathbb{Q}} \mathbb{E}^{\mathbb{Q}}\left[D_{t_{N-n+1}}\right], \sup _{\mathbb{Q}} \mathbb{E}^{\mathbb{Q}}\left[D_{t_{N-n+1}}\right]\right],
$$


where $\mathbb{Q}$ is the set of probability measures. Thus

$$
\begin{array}{r}
\Delta D_{t_{N-n}}(\omega) \in\left[D_{t_{N-n+1}}-\sup _{\mathbb{Q}} \mathbb{E}^{\mathbb{Q}}\left[D_{t_{N-n+1}}\right],\right. \\
\left.D_{t_{N-n+1}}-\inf _{\mathbb{Q}} \mathbb{E}^{\mathbb{Q}}\left[D_{t_{N-n+1}}\right]\right],
\end{array}
$$

so the sets $\left\{\omega: \Delta D_{t_{N-n}}(\omega)>0\right\}$ and $\left\{\omega: \Delta D_{t_{N-n}}(\omega)<0\right\}$ have positive probability. Consequently, it follows that

$$
g_{N-n}(\alpha, \infty, \cdot)=\infty ; \quad g_{N-n}(\alpha,-\infty, \cdot)=\infty
$$

Therefore the minimum of $g_{N-n}(\alpha, \beta, \cdot)$ is a critical point. Hence

$$
\begin{aligned}
\frac{\partial g_{N-n}}{\partial \alpha}= & \left.\frac{\left(\mu_{t_{N-n}}^{c} h+\sigma_{t_{N-n}}^{c} \sqrt{h}\right)}{2} e^{-\gamma_{t_{N-n}} \alpha\left(\mu_{t_{N-n}}^{c} h+\sigma_{t_{N-n}}^{c}\right.} \sqrt{h}\right) \\
& \times \mathbb{E}_{t_{N-n}}\left[e^{-\gamma_{t_{N-n}} \beta^{*} \Delta D_{t_{N-n}}} e^{-\gamma_{t_{N-n}} Y_{t_{N-n+1}}^{*}} \mid A_{t_{N-n}}\right] \\
& +\frac{\left(\mu_{t_{N-n}}^{c} h-\sigma_{t_{N-n}}^{c} \sqrt{h}\right)}{2} e^{-\gamma_{t_{N-n}} \alpha\left(\mu_{t_{N-n}}^{c} h-\sigma_{t_{N-n}}^{c} \sqrt{h}\right)} \\
& \times \mathbb{E}_{t_{N-n}}\left[e^{-\gamma_{t_{N-n}} \beta^{*} \Delta D_{t_{N-n}}} e^{-\gamma_{t_{N-n}} Y_{t_{N-n+1}}^{*}} \mid A_{t_{N-n}}^{c}\right] \\
= & 0 .
\end{aligned}
$$

By direct calculation, we verify that the subgame perfect strategy is

$$
\begin{aligned}
& \alpha_{t_{N-n}}^{*} \\
& =\frac{1}{2 \gamma_{t_{N-n}} \sigma_{t_{N-n}}^{c} \sqrt{h}} \log \left(\frac{1+r_{t_{N-n}}^{c} \sqrt{h}}{1-r_{t_{N-n}}^{c} \sqrt{h}}\right) \\
& \quad+\frac{1}{2 \gamma_{t_{N-n}} \sigma_{t_{N-n}}^{c} \sqrt{h}} \\
& \quad \times \log \left(\frac{\mathbb{E}_{t_{N-n}}\left[e^{-\gamma_{t_{N-n}} \beta_{t_{N-n}}^{*} D_{t_{N-n+1}}} e^{-\gamma_{t_{N-n}} Y_{t_{N-n+1}}^{*}} \mid A_{t_{N-n}}\right]}{\mathbb{E}_{t_{N-n}}\left[e^{-\gamma_{t_{N-n}} \beta_{t_{N-n}}^{*} D_{t_{N-n+1}}} e^{\left.-\gamma_{t_{N-n}} Y_{t_{N-n+1}}^{*} \mid A_{t_{N-n}}^{c}\right]}\right.}\right) .
\end{aligned}
$$

From the equilibrium conditions it follows that $\beta_{t_{N-n}}^{*}=1$. This, combined with $\partial g_{N-n} / \partial \beta=0$, yields the equilibrium price at $T_{N-n}$. First, from $\partial g_{N-n} / \partial \beta=0$, one gets

$$
\begin{aligned}
\mathbb{E}_{t_{N-n}}[ & \left.\Delta D_{t_{N-n}} e^{-\gamma_{t_{N-n}} m_{D} \Delta D_{t_{N-n}}} e^{-\gamma_{t_{N-n}} Y_{t_{N-n+1}}^{*}} \mid A_{t_{N-n}}\right] \\
= & -e^{2 \gamma_{t_{N-n}} \alpha_{t_{N-n}}^{*} \sigma_{t_{N-n}}^{c} \sqrt{h}} \mathbb{E}_{t_{N-n}} \\
& \times\left[\Delta D_{t_{N-n}} e^{-\gamma_{t_{N-n}} m_{D} \Delta D_{t_{N-n}}} e^{-\gamma_{t_{N-n}} Y_{t_{N-n+1}}^{*}} \mid A_{t_{N-n}}^{c}\right] .
\end{aligned}
$$

From $\partial g_{N-n} / \partial \alpha=0$ it follows that

$$
\begin{aligned}
& e^{2 \gamma_{t_{N-n}} \alpha_{t_{N-n}}^{*} \sigma_{t_{N-n}}^{c} \sqrt{h}} \\
& =\left(\left(1+r_{t_{N-n}}^{c} \sqrt{h}\right) \mathbb{E}_{t_{N-n}}\right. \\
& \left.\times\left[e^{-\gamma_{t_{N-n}} m_{D} D_{t_{N-n+1}}} e^{-\gamma_{t_{N-n}} Y_{t_{N-n+1}^{*}}^{*}} \mid A_{t_{N-n}}\right]\right) \\
& \times\left(\left(1-r_{t_{N-n}}^{c} \sqrt{h}\right) \mathbb{E}_{t_{N-n}}\right. \\
& \left.\times\left[e^{-\gamma_{t_{N-n}} m_{D} D_{t_{N-n+1}}} e^{-\gamma_{t_{N-n}} Y_{t_{N-n+1}}^{*}} \mid A_{t_{N-n}}^{c}\right]\right)^{-1} .
\end{aligned}
$$

This, together with

$$
\Delta D_{t_{N-n}}=D_{t_{N-n+1}}-\mathbb{E}_{t_{N-n}}^{\mathbb{Q}^{*}}\left[D_{t_{N-n+1}}\right],
$$

(here $\mathbb{Q}^{*}$ is the equilibrium probability measure to be found) yields

$$
\begin{gathered}
\frac{2}{1-r_{t_{N-n}}^{c} \sqrt{h}} \mathbb{E}_{t_{N-n}^{Q^{*}}}^{Q^{*}}\left[D_{t_{N-n+1}}\right] \\
=\left(\mathbb { E } _ { t _ { N - n } } \left[D_{t_{N-n+1}} e^{-\gamma_{t_{N-n}} m_{D} D_{t_{N-n+1}}}\right.\right. \\
\left.\left.\times e^{-\gamma_{t_{N-n}} Y_{t_{N-n+1}}^{*} \mid} \mid A_{t_{N-n}}\right]\right) \\
\times\left(\mathbb { E } _ { t _ { N - n } } \left[e^{-\gamma_{t_{N-n}} m_{D} D_{t_{N-n+1}}}\right.\right. \\
\left.+\frac{\left(1+r_{t_{N-n}}^{c}\right.}{\left(1-r_{t_{N-n}}^{c}\right.} \sqrt{h}\right) \\
\times\left(\mathbb{E}_{t_{N-n}}\left[D_{t_{N-n+1}} e^{-\gamma_{t_{N-n}} Y_{t_{N-n+1}}^{*} m_{D} D_{t_{N-n+1}}} \mid A_{t_{N-n}}\right]\right)^{-1} \\
\times\left(\mathbb { E } _ { t _ { N - n } } \left[e^{-\gamma_{t_{N-n}} m_{D} D_{t_{N-n+1}}}\right.\right. \\
\left.\left.\left.\times e^{-\gamma_{t_{N-n}} Y_{t_{N-n+1}}^{*}} \mid A_{t_{N-n}}^{c}\right] Y_{t_{N-n+1}}^{*} \mid A_{t_{N-n}}^{c}\right]\right)^{-1} .
\end{gathered}
$$

Consequently

$$
\begin{aligned}
& D_{t_{N-n}} \\
& =\frac{1-r_{t_{n}}^{c} \sqrt{h}}{2} \\
& \quad \times\left(\mathbb { E } _ { t _ { N - n } } \left[D_{t_{N-n+1}} e^{-\gamma_{t_{N-n}} m_{D} D_{t_{N-n+1}}}\right.\right. \\
& \left.\left.\quad \times e^{-\gamma_{t_{N-n}} Y_{t_{N-n+1}}^{*}} \mid A_{t_{N-n}}\right]\right) \\
& \times\left(\mathbb { E } _ { t _ { N - n } } \left[e^{-\gamma_{t_{N-n}} m_{D} D_{t_{N-n+1}}}\right.\right. \\
& \left.\left.\quad \times e^{-\gamma_{t_{N-n}} Y_{t_{N-n+1}}^{*}} \mid A_{t_{N-n}}\right]\right)^{-1}
\end{aligned}
$$




$$
\begin{gathered}
+\frac{1+r_{t_{n}}^{c} \sqrt{h}}{2} \\
\times\left(\mathbb { E } _ { t _ { N - n } } \left[D_{t_{N-n+1}} e^{-\gamma_{t_{N-n}} m_{D} D_{t_{N-n+1}}}\right.\right. \\
\left.\left.\times e^{-\gamma_{t_{N-n}} Y_{t_{N-n+1}}^{*}} \mid A_{t_{N-n}}^{c}\right]\right) \\
\times\left(\mathbb { E } _ { t _ { N - n } } \left[e^{-\gamma_{t_{N-n}} m_{D} D_{t_{N-n+1}}}\right.\right. \\
\left.\left.\times e^{-\gamma_{t_{N-n}} Y_{t_{N-n+1}}^{*}} \mid A_{t_{N-n}}^{c}\right]\right)^{-1} .
\end{gathered}
$$

Thus, the equilibrium price is

$$
D_{t_{N-n}}=\mathbb{E}_{t_{N-n}}\left[D_{t_{N-n+1}} \Lambda_{t_{N-n+1}}^{*}\right],
$$

with $\Lambda_{t_{N-n+1}}^{*}$ defined in (35).

\section{Proof of Corollary 9}

We consider the time period $[(N-n) h,(N-n+1) h)$. Recall that

$$
\widehat{X}_{t_{N}}=\widehat{X}_{t_{N-n}}+\Delta \widehat{X}_{t_{N-n}}+\sum_{k=N-(n-1)}^{N-1} \Delta \widehat{X}_{t_{k}}
$$

where

$$
\Delta \widehat{X}_{t_{k}}=\widehat{\alpha}_{t_{k}}\left(\mu_{t_{k}}^{c} h+\sigma_{t_{k}}^{c} \sqrt{h} \Delta b_{t_{k}}^{1}\right)+m_{D} \Delta D_{t_{k}},
$$

for $k=N-(n-1), \ldots, N-2, N-1$. By definition of $\widehat{Y}_{t_{k}}$, we have

$$
\begin{aligned}
& \mathbb{E}_{t_{N-n}}\left[U^{\prime}\left(\widehat{W}_{t_{N}}\right)\right]=\gamma_{t_{0}} e^{-\gamma_{t_{0}} \widehat{X}_{t_{N-n}}} \mathbb{E}_{t_{N-n}}\left[e^{-\gamma_{t_{0}} \Delta \widehat{X}_{t_{N-n}}} e^{-\gamma_{t_{0}} \widehat{\widehat{t}}_{t_{N-n+1}}}\right], \\
& \mathbb{E}_{t_{N-n+1}}\left[U^{\prime}\left(\widehat{W}_{t_{N}}\right)\right]=\gamma_{t_{0}} e^{-\gamma_{t_{0}} \widehat{X}_{t_{N}-n}} e^{-\gamma_{t_{0}} \Delta \widehat{X}_{t_{N-n}}} e^{-\gamma_{t_{0}} \widehat{Y}_{t_{N-n+1}}}, \\
& \frac{\mathbb{E}_{t_{N-n+1}}\left[U^{\prime}\left(\widehat{W}_{t_{N}}\right)\right]}{\mathbb{E}_{t_{N-n}}\left[U^{\prime}\left(\widehat{W}_{t_{N}}\right)\right]} \\
& =\frac{e^{-\gamma_{t_{0}} \Delta \widehat{X}_{t_{N-n}}} e^{-\gamma_{t_{0}} \widehat{Y}_{t_{N-n+1}}}}{\mathbb{E}_{t_{N-n}}\left[e^{-\gamma_{t_{0}} \Delta \widehat{X}_{t_{N-n}}} e^{-\gamma_{t_{0}} \widehat{Y}_{t_{N-n+1}}}\right]}
\end{aligned}
$$

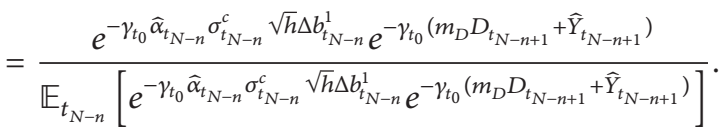

Recall that

$$
\begin{aligned}
& e^{2 \gamma_{t_{0}} \widehat{\bar{t}}_{t_{N-n}} \sigma_{t_{N-n}}^{c} \sqrt{h}} \\
& =\frac{\left(1+r_{t_{N-n}}^{c}\right) \mathbb{E}_{t_{N-n}}\left[e^{-\gamma_{t_{0}}\left(m_{D} D_{t_{N-n+1}}+\widehat{Y}_{t_{N-n+1}}\right)} \mid A_{t_{N-n}}\right]}{\left(1-r_{t_{N-n}}^{c}\right) \mathbb{E}_{t_{N-n}}\left[e^{-\gamma_{t_{0}}\left(m_{D} D_{t_{N-n+1}}+\widehat{Y}_{t_{N-n+1}}\right)} \mid A_{t_{N-n}}^{c}\right]} .
\end{aligned}
$$

Using (C.4), we have

$$
\begin{aligned}
\mathbb{E}_{t_{N-n}}\left[e^{-\gamma_{t_{0}} \widehat{\alpha}_{t_{N-n}} \sigma_{t_{N-n}}^{c} \sqrt{h} \Delta b_{t_{N-n}}^{1}}\right. & \left.\times e^{-\gamma_{t_{0}}\left(m_{D} D_{t_{N-n+1}}+\widehat{Y}_{t_{N-n+1}}\right)}\right] \\
= & \frac{1}{2} e^{-\gamma_{t_{0}} \widehat{\alpha}_{t_{N-n}} \sigma_{t_{N-n}}^{c} \sqrt{h}} \mathbb{E}_{t_{N-n}} \\
& \times\left[e^{-\gamma_{t_{0}}\left(m_{D} D_{t_{N-n+1}}+\widehat{Y}_{t_{N-n+1}}\right)} \mid A_{t_{N-n}}\right] \\
& +\frac{1}{2} e^{\gamma_{t_{0}} \widehat{\alpha}_{t_{N-n}} \sigma_{t_{N-n}}^{c} \sqrt{h}} \mathbb{E}_{t_{N-n}} \\
& \times\left[e^{-\gamma_{t_{0}}\left(m_{D} D_{t_{N-n+1}}+\widehat{T}_{t_{N-n+1}}\right)} \mid A_{t_{N-n}}^{c}\right] \\
= & \frac{1}{1+r_{t_{N-n}}^{c} \sqrt{h}} e^{\gamma_{t_{0}} \widehat{\alpha}_{t_{N-n}} \sigma_{t_{N-n}}^{c} \sqrt{h}} \mathbb{E}_{t_{N-n}} \\
& \times\left[e^{-\gamma_{t_{0}}\left(m_{D} D_{t_{N-n+1}}+\widehat{T}_{t_{N-n+1}}\right)} \mid A_{t_{N-n}}^{c}\right] \\
= & \frac{1}{1-r_{t_{N-n}}^{c} \sqrt{h}} e^{-\gamma_{t_{0}} \widehat{\alpha}_{t_{N-n}} \sigma_{t_{N-n}}^{c} \sqrt{h}} \mathbb{E}_{t_{N-n}} \\
& \times\left[e^{-\gamma_{t_{0}}\left(m_{D} D_{t_{N-n+1}}+\widehat{Y}_{t_{N-n+1}}\right)} \mid A_{t_{N-n}}\right] .
\end{aligned}
$$

Therefore, it follows that on the set of $\left\{\omega: \omega \in A_{t_{N-n}}^{c}\right\}$

$$
\begin{aligned}
& \frac{\mathbb{E}_{t_{N-n+1}}\left[U^{\prime}\left(\widehat{W}_{t_{N}}\right)\right]}{\mathbb{E}_{t_{N-n}}\left[U^{\prime}\left(\widehat{W}_{t_{N}}\right)\right]} \\
& =\frac{\left(1+r_{t_{N-n}}^{c} \sqrt{h}\right) e^{-\gamma_{t_{0}}\left(m_{D} D_{t_{N-n+1}}+\widehat{Y}_{t_{N-n+1}}\right)}}{\mathbb{E}_{t_{N-n}}\left[e^{-\gamma_{t_{0}}\left(m_{D} D_{t_{N-n+1}}+\widehat{Y}_{t_{N-n+1}}\right)} \mid A_{t_{N-n}}^{c}\right]} .
\end{aligned}
$$

Moreover, on the set of $\left\{\omega: \omega \in A_{t_{\mathrm{N}-n}}\right\}$,

$$
\begin{aligned}
\frac{\mathbb{E}_{t_{N-n+1}}\left[U^{\prime}\left(\widehat{W}_{t_{N}}\right)\right]}{\mathbb{E}_{t_{N-n}}\left[U^{\prime}\left(\widehat{W}_{t_{N}}\right)\right]} \\
=\frac{\left(1-r_{t_{N-n}}^{c} \sqrt{h}\right) e^{-\gamma_{t_{0}}\left(m_{D} D_{t_{N-n+1}}+\widehat{Y}_{t_{N-n+1}}\right)}}{\mathbb{E}_{t_{N-n}}\left[e^{-\gamma_{t_{0}}\left(m_{D} D_{t_{N-n+1}}+\widehat{Y}_{t_{N-n+1}}\right)} \mid A_{t_{N-n}}\right]} .
\end{aligned}
$$

Therefore

$$
\frac{\mathbb{E}_{t_{N-n+1}}\left[U^{\prime}\left(\widehat{W}_{t_{N}}\right)\right]}{\mathbb{E}_{N-n}\left[U^{\prime}\left(\widehat{W}_{t_{N}}\right)\right]}=\widehat{\Lambda}_{t_{N-n+1}},
$$

with $\widehat{\Lambda}_{t_{N-n+1}}$ defined in (36).

\section{Conflict of Interests}

The authors declare that there is no conflict of interests regarding the publication of this paper. 


\section{Acknowledgments}

This work is supported by NSERC Grants 371653-09 and 88051 and MITACS Grants 5-26761 and 30354 and by the Natural Science Foundation of China (10901086). The authors would like to thank Tom Hurd for helpful comments.

\section{References}

[1] M. Rubinstein, "The valuation of uncertain income streams and the pricing of options," The Rand Journal of Economics, vol. 7, no. 2, pp. 407-425, 1976.

[2] M. J. Brennan, "The pricing of contingent claims in discrete time models," The Journal of Finance, vol. 34, no. 1, pp. 53-68, 1979.

[3] S. Bhattacharya, "Notes on multiperiod valuation and the pricing of options," The Journal of Finance, vol. 36, no. 1, pp. 163$180,1981$.

[4] A. Bizid and E. Jouini, "Incomplete markets and short-sales constraints: an equilibrium approach," International Journal of Theoretical and Applied Finance, vol. 4, no. 2, pp. 211-243, 2001.

[5] A. Câmara, "A generalization of the brennan-rubinstein approach for the pricing of derivatives," The Journal of Finance, vol. 58 , no. 2, pp. 805-819, 2003.

[6] M. Cao and J. Wei, "Weather derivatives valuation and market price of weather risk," Journal of Futures Markets, vol. 24, no. 11, pp. 1065-1089, 2004.

[7] Y. Lee and S. S. Oren, "An equilibrium pricing model for weather derivatives in a multi-commodity setting," Energy Economics, vol. 31, no. 5, pp. 702-713, 2009.

[8] Y. Lee and S. S. Oren, "A multi-period equilibrium pricing model of weather derivatives," Energy Systems, vol. 1, no. 1, pp. 3-30, 2010.

[9] P. Cheridito, U. Horst, M. Kupper, and T. A. Pirvu, "Equilibrium pricing in incomplete markets under translation invariant preferences," 2012, http://ssrn.com/abstract=1755610 .

[10] R. E. Lucas, Jr., "Asset prices in an exchange economy," Econometrica, vol. 46, no. 6, pp. 1429-1445, 1978.

[11] S. Hodges and A. Neuberger, "Optimal replication of contingent claims under transaction costs," Review of Futures Markets, vol. 8, pp. 222-239, 1989.

[12] M. Musiela, E. Sokolova, and T. Zariphopoulou, "Indifference valuation in incomplete binomial models," Mathematics in Action, vol. 3, no. 2, pp. 1-36, 2010.

[13] V. Henderson, "Valuation of claims on nontraded assets using utility maximization," Mathematical Finance, vol. 12, no. 4, pp. 351-373, 2002.

[14] M. Musiela and T. Zariphopoulou, "A valuation algorithm for indifference prices in incomplete markets," Finance and Stochastics, vol. 8, no. 3, pp. 399-414, 2004.

[15] V. Henderson and D. Hobson, "Utility indierence pricing-an overview," in Volume on Indierence Pricing, R. Carmona, Ed., Princeton University Press, Princeton, NJ, USA, 2004.

[16] T. A. Pirvu and H. Zhang, "Utility indierence pricing: a time consistent approach," Applied Mathematical Finance, vol. 20, pp. 304-326, 2013.

[17] S. Gordon and P. St-Amour, "A preference regime model of bull and bear markets," American Economic Review, vol. 90, no. 4, pp. 1019-1033, 2000.

[18] N. Barberis and M. Huang, "Mental accounting, loss aversion, and individual stock returns," The Journal of Finance, vol. 56, no. 4, pp. 1247-1292, 2001.
[19] J.-P. Danthine, J. B. Donaldson, C. Giannikos, and H. Guirguis, "On the consequences of state dependent preferences for the pricing of financial assets," Finance Research Letters, vol. 1, no. 3, pp. 143-153, 2004.

[20] S. Gordon and P. St-Amour, "Asset returns and state-dependent risk preferences," Journal of Business \& Economic Statistics, vol. 22, no. 3, pp. 241-252, 2004.

[21] B. Yuan and K. Chen, "Impact of investor's varying risk aversion on the dynamics of asset price fluctuations," Journal of Economic Interaction and Coordination, vol. 1, no. 2, pp. 189-214, 2006.

[22] T. Berrada, J. Detemple, and M. Rindisbacher, "Asset pricing with regime-dependent preferences and learning," 2013, http:// ssrn.com/abstract $=2313807$.

[23] T. Björk and A. Murgoci, "A general theory of Markovian time inconsistent stochastic control problems," 2010, http://ssrn .com/abstract $=1694759$.

[24] H. Wu, "Time-consistent strategies for a multiperiod meanvariance portfolio selection problem," Journal of Applied Mathematics, vol. 2013, Article ID 841627, 13 pages, 2013.

[25] N. Gârleanu, L. H. Pedersen, and A. M. Poteshman, "Demandbased option pricing," Review of Financial Studies, vol. 22, no. 10, pp. 4259-4299, 2009. 


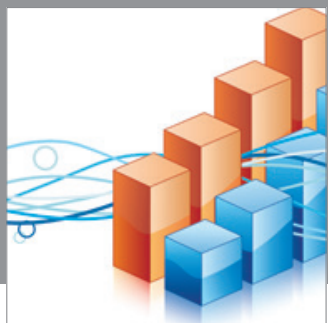

Advances in

Operations Research

mansans

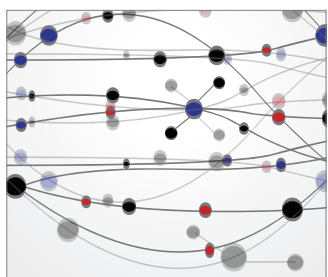

The Scientific World Journal
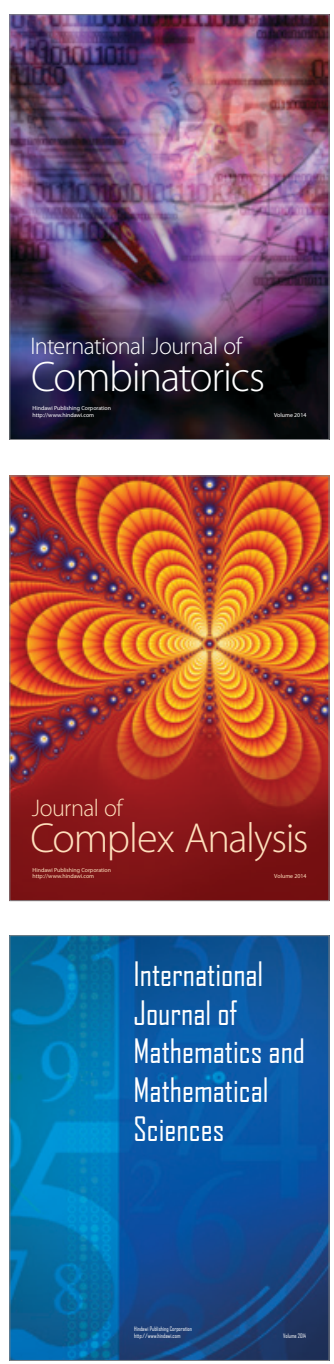
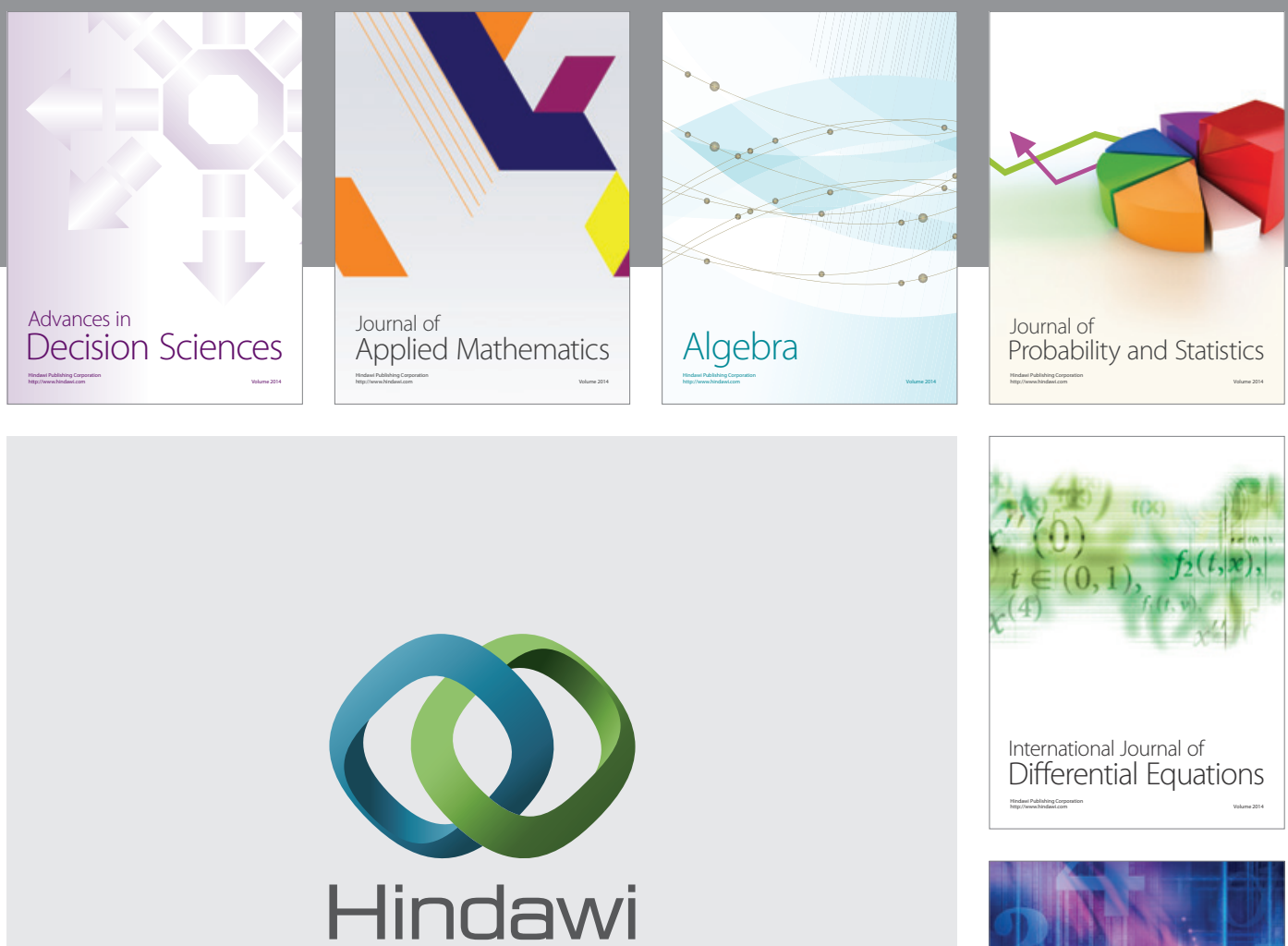

Submit your manuscripts at http://www.hindawi.com
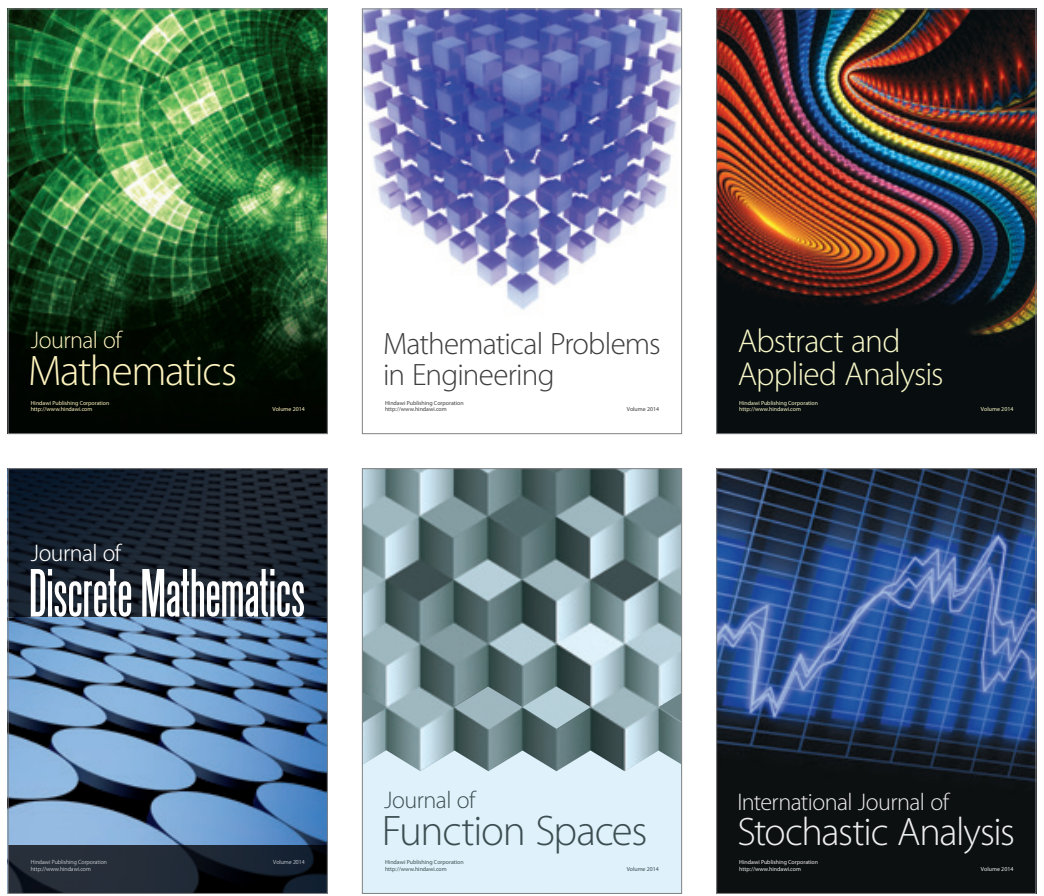

Journal of

Function Spaces

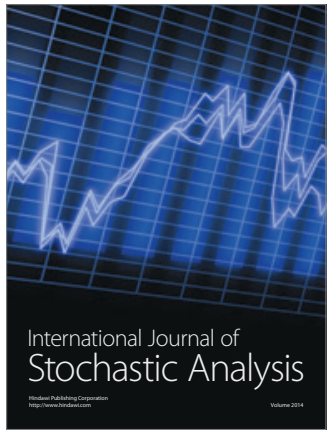

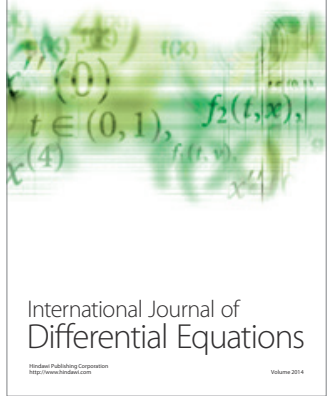
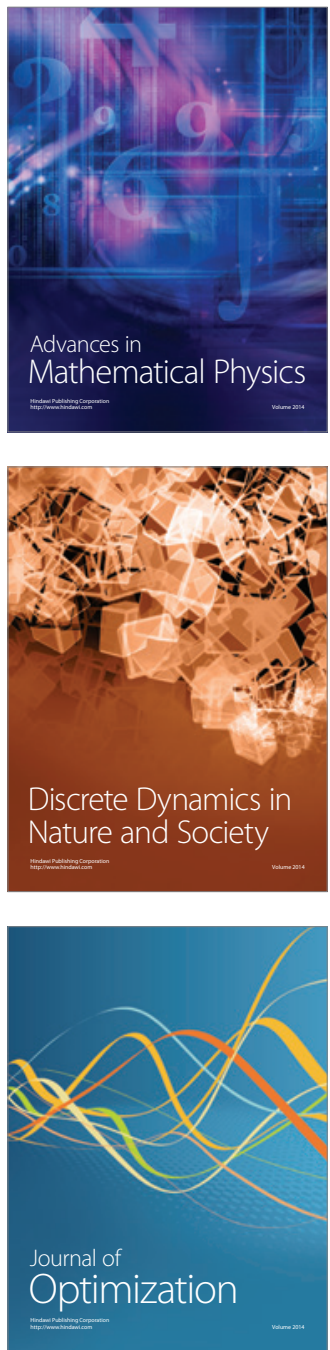